\title{
Techniques for ventricular repolarization instability assessment from the ECG
}

\author{
Pablo Laguna, Juan Pablo Martínez, Esther Pueyo
}

\begin{abstract}
Instabilities in ventricular repolarization have been documented to be tightly linked to arrhythmia vulnerability. Translation of the information contained in the repolarization phase of the ECG into valuable clinical decision-making tools remains challenging. This work aims at providing an overview of the last advances in the proposal and quantification of ECG-derived indices that describe repolarization properties and whose alterations are related with threatening arrhythmogenic conditions. A review of the state-of-the-art is provided, spanning from the electrophysiological basis of ventricular repolarization to its characterization on the surface ECG through a set of temporal and spatial risk markers.
\end{abstract}

Index Terms-Electrophysiological basis of the ECG, ECG waves, ECG intervals, repolarization instabilities, spatial and temporal ventricular repolarization dispersion, cardiac arrhythmias, biophysical modeling of the ECG, ECG signal processing, repolarization risk markers, $T$ wave alternans, $Q T$ variability.

\section{INTRODUCTION}

Since its invention by Willem Einthoven (1860-1927) the electrocardiogram (ECG) has become the most widely-used tool for cardiac diagnosis. The ECG describes the electrical activity of the heart, as recorded by electrodes placed on the body surface. This activity is the summed result of the different action potentials (APs), concurring simultaneously, from all excitable cells throughout the heart as they trigger contraction. The trace of each heartbeat in the ECG signal consists on a sequence of characteristic deflections or waves, whose morphology and timing convey useful information to identify disturbances in the heart's electrical activity.

The timing of successive heartbeats [1] or wave shape patterns, the coupled relationship between parameters associated with those patterns, their evolution over time, their responses to heart rate changes, their spatial distribution, etc, may provide useful information about the underlying physiological phenomena under study, which become the driving forces for the methodological developments of ECG signal processing [2].

The lead system, or body locations where the electrodes are located for ECG acquisition, are usually standardized.

\section{Asterisk indicates corresponding author.}

This project was support by projects TEC2013-42140-R and TIN201341998-R from CICYT/FEDER Spain, and from Aragón Government, Spain and from European Social Fund (EU) through Biomedical Signal Interpretation and Computational Simulation (BSICoS) group ref:T96. The computation was performed by the ICTS 0707NANBIOSIS, by the High Performance Computing Unit of the CIBER in Bioengineering, Biomaterials \& Nanomedicine (CIBER-BBN) at the University of Zaragoza.

P. Laguna, E. Pueyo and J.P. Martínez are with the BSICoS Group, Aragón Institute of Engineering Research, University of Zaragoza, Zaragoza 50015, and Centro de Investigacioón Biomédica en Red en Bioingeniería, Biomateriales y Nanomedicina (CIBER-BBN), Madrid, Spain (e-mail: \{laguna,epueyo,jpmart\}@unizar.es)

PL, on a personal note, acknowledges the stimulus of Isabel, on behalf of the research for disease cures, and so overcoming several difficulties which led this paper to be ruled out several times during writing.
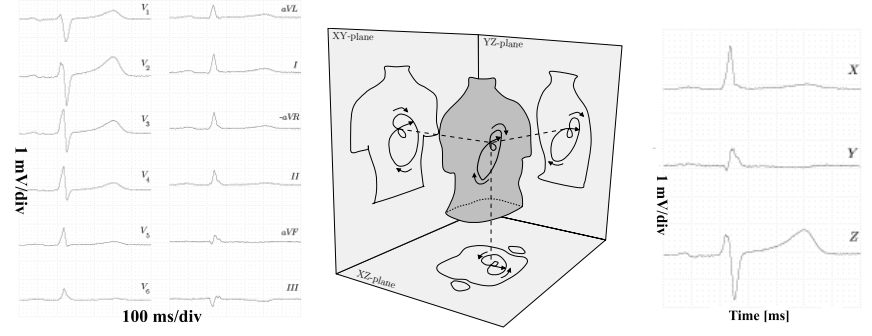

Fig. 1. Left panel, the standard 12-lead ECG. The ECG corresponds to a healthy subject. Central panel: a vectorcardiographic loop and its projection onto the three orthogonal planes. Right panel: the orthogonal vectorcardiographic leads. Adapted from [3].

This facilitates inter-subject and serial comparison of measurements. The conventional lead systems are the 12-lead system, typically used for recordings at rest, and the orthogonal lead system, whose three leads jointly form the vectorcardiogram (VCG), and which can be either directly recorded or derived from the 12 standard leads, see Fig. 1. There is a large bibliography dealing with the basis of electrocardiography [1] and basis combined with signal processing [3]. In addition to resting ECG, several other lead systems, depending on the purpose of the exploration, can be found. To name some, we refer to intensive care monitoring, ambulatory monitoring, stress test, high resolution ECG, polysomnographic recordings, etc.

The ECG can be viewed as spatio-temporal integration of the APs associated with all of the cardiac cells [3], [4] (see Fig. 2). Fig. 3 shows a cardiac cycle, illustrating the most relevant ECG waves. The $\mathrm{T}$ wave is the one that reflects ventricular repolarization. Instabilities in ventricular repolarization have been documented to be tightly linked to arrhythmia development [5], thus justifying the interest in the analysis and review of methods dealing with $\mathrm{T}$ wave characterization and quantification. The present paper follows from a previous review on cardiac repolarization analysis by the same authors [6].

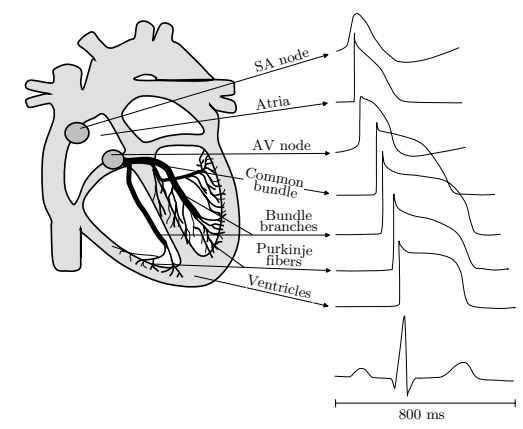

Fig. 2. Morphology and timing of APs from different regions of the heart and the related cardiac cycle of the ECG. Adapted from [3].

Sudden cardiac death (SCD) is a major cause of death 


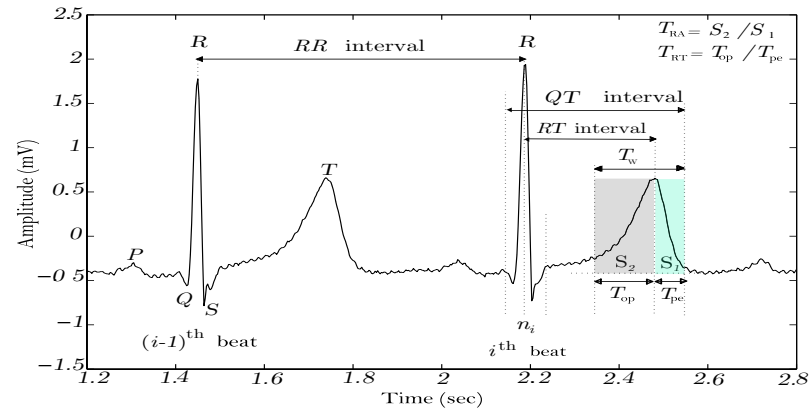

Fig. 3. ECG of two cardiac cycles and most relevant intervals and waves.

in developed countries, where 1 out of 1000 subjects die every year due to SCD [7]. This is about $20 \%$ of all deaths, which underscores the importance of its prevention [8]. Ventricular arrhythmias, such as ventricular tachycardia (VT) or ventricular fibrillation (VF), are the cause of most SCDs [9], whereas only a small percentage of cases of SCD are due to bradycardia.

Three main factors have been identified to have a major role in the initiation and maintenance of arrhythmias: substrate, triggers and modulators. A vulnerable myocardium is the substrate for arrhythmogenesis, meaning that when triggering factors appear, they can lead to malignant arrhythmias potentially ending in SCD. Increased dispersion of the repolarization properties among different ventricular myocardial cells or regions has been identified as a characteristic of a vulnerable substrate [10]. Other factors can modulate the arrhythmogenic substrate or the triggers by altering the electrophysiological properties of the heart. An important modulator is the autonomic nervous system (ANS) [11].

Therapeutic choices designed to treat cardiac arrhythmias, and eventually prevent SCD, are highly conditioned by the factors (substrate, triggers and modulators) that contribute to their generation. Implantable cardioverter defibrillators (ICDs) are designed to apply an electric shock to the heart in the presence of VT or VF and restore its sinus rhythm. Antiarrhythmic drugs, by acting on some of those factors, prevent the occurence of arrhythmias, thus reducing the probability of SCD. The use of these therapies (or a combination of them) must be assessed in terms of safety for the patient and costeffectiveness. This justifies the importance and necessity of developing strategies to identify high-risk patients who would benefit from a specific therapy.

Repolarization analysis based on the ECG is a low-cost, non-invasive approach that has been shown to be useful for risk assessment [6] and can be applied to the general population. Currently, challenges in this matter involve better understanding of the electrophysiological bases responsible for or secondary to the development of an arrhythmogenic substrate. When this better knowledge is paired with better understanding of the transformation from cellular electrical activity to surface ECG, then better targeted ECG-based risk stratification markers may become available.

In section II-A of this paper, the ionic and cellular bases of ECG repolarization patterns under physiological conditions are presented and in section II-B, under pathological conditions as the basis for translation of cellular signatures to the surface ECG. In section II-C a method for biophysical representation of tissue properties and its correspondence into the ECG [12] is also presented, which can be useful when global myocardium property distributions are in need for ECG interpretation and risk identification. In section III-A basic concepts of ECG signal processing are described. In section III-B ECG features characterizing the spatial variation of repolarization are reviewed. Section III-C explores ECG measurements and morphological markers describing temporal variability of ventricular repolarization, including the dynamics of QT dependence on heart rate (HR). Section III-D introduces $\mathrm{T}$ wave alternans and other novel ECG indices integrating spatial and temporal dynamics of ventricular repolarization. Challenges and future perspectives on ECG-based repolarization assessment are presented in section IV. Finally, conclusions are presented in section $\mathrm{V}$.

\section{ELECTROPHYSIOLOGICAL BASIS OF REPOLARIZATION INSTABILITIES}

\section{A. Repolarization waveforms}

1) Membrane currents and AP: Establishing a relationship between ECG and AP properties can prove fundamental for a better understanding of the mechanisms underlying cardiac arrhythmias. The AP associated with each cardiac cell is the result of ion charges moving in and out of the cell through voltage-gated channels. A representative AP of a ventricular myocyte is presented in Fig. 4a. Phases 0-4 in the AP can be appreciated, with different currents through ion channels and electrogenic transporters contributing to each of them (Fig. 4b). Some of those currents are notably differently expressed across the ventricles.

In the last years, mathematical models have been proposed to describe electrical and ionic homeostasis in human ventricular myocytes. A relevant model of human ventricular AP was proposed by Iyer et al. [13], reproducing diverse aspects of the excitationcontraction coupling. One of the most widely used models is the one proposed by ten Tusscher \& Panfilov [14]. Later, another model of human ventricular AP was proposed by Grandi et al. [15], which was subsequently modified to accurately reproduce arrhythmic risk markers recorded in experiments [16]. The O'Hara et al. model is the most upto-date model of a human ventricular myocyte [17].

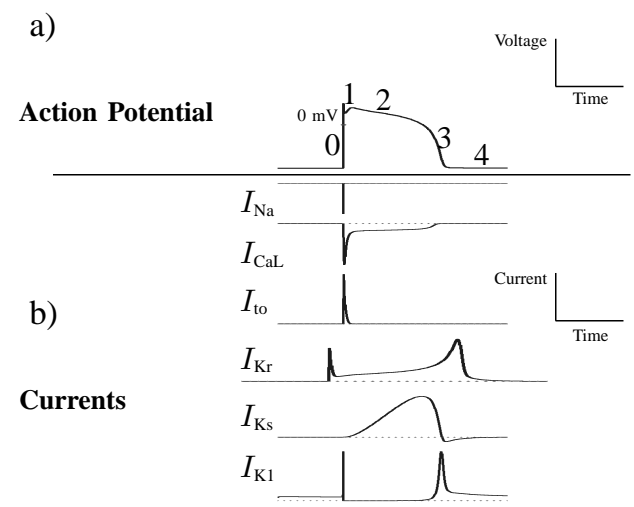

Fig. 4. a) Action potential of a ventricular myocyte, with indication of its phases. b) Ionic currents underlying the different AP phases are illustrated. 


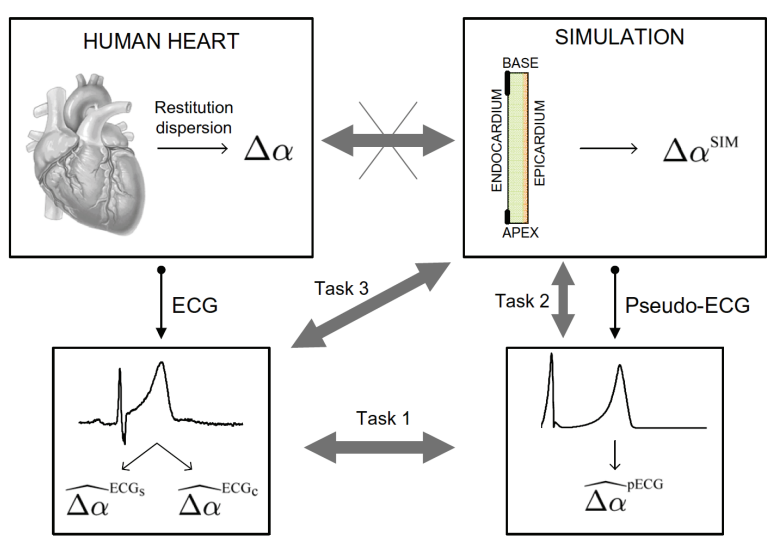

Fig. 5. Outline of a simulation, where the recorded ECG, the simulated Pseudo-ECG, the real myocardium and a simulated setup, are used for comparison of simulated and recorder markers (see section III-D2). In this case a 2D tissue slice with a particular cell type distribution across the ventricular wall is used. Crossed arrow shows a desirable but unaccessible connection. Tasks 1, 2 and 3 represent the different comparison tasks that can be done. Reproduced from [20].

2) Intrinsic heterogeneities: Differences in repolarizing currents have been documented between anterior, inferior and posterior walls of the left ventricle, and also between apex and base [18]. Transmural differences exist as well, with endocardial, midmyocardial and epicardial cells having been described. Most mathematical models of human ventricular electrophysiology account for such heterogeneities. Intrinsic ventricular heterogeneities are essential for cardiac function under normal physiological conditions.

3) Genesis of ECG repolarization waves and intervals: The $\mathrm{T}$ wave of the ECG reflects heterogeneities in ventricular repolarization, see Fig. 2. Its formation depends both on the sequence of ventricular activation and on the heterogeneities in AP characteristics throughout ventricular myocardium [19]. The QT interval of the ECG, measured from QRS onset to $\mathrm{T}$ wave end, has been used in most repolarization studies. It represents the time needed for ventricular depolarization plus repolarization and it is closely related to the AP duration (APD) of ventricular cells.

From the knowledge of the electrical activity at the cellular and tissue levels, one can approach the issue of simulating ECG signals based on a specified spatial distribution of cells within the myocardium and considering a particular excitation pattern. 1D, 2D or 3D tissue models can be generated, where geometry, anisotropy, connectivity, propagation velocity etc, need to be taken into account. Additionally, modeling of the torso leads to more accurate simulation of surface ECG signals. One schematic example of this process can be seen in Fig. 5.

\section{B. Abnormal repolarization and cardiac arrhythmias}

1) Pathological heterogeneities: Many cardiac pathologies accentuate intrinsic heterogeneities in ventricular repolarization. Pathological states associated with enhanced repolarization heterogeneities include ischemia, Brugada syndrome, long QT syndrome (LQTS) or heart failure. In section III-B1 methods to quantify dispersion of repolarization from surface
ECG time intervals will be described. In section III-B2 methods used to evaluate electrocardiographic $\mathrm{T}$ wave morphology changes, as a reflection of amplified heterogeneities in AP repolarization, will be presented.

In addition to spatial heterogeneities, increased temporal repolarization heterogeneities have been as well linked to proarrhythmia. A phenomenon associated with temporal repolarization heterogeneity is abnormal APD adaptation in response to cycle length changes, which has been suggested to play a role in the genesis of arrhythmias [21]. Evaluation of ECG repolarization adaptation to HR changes will be presented in section III-C1. Another phenomenon is AP variability, measured as fluctuations in the duration of the AP, which has been closely linked to SCD under different conditions [22]. QT variability quantified from the surface ECG can be considered as an approximation to the study of such phenomenon and it will be explored in section III-C2. Section III-D1 will examine T wave alternans (TWA), which have been shown to be proarrhythmic in different investigations [23]. TWA is considered as a manifestation of spatial or temporal dispersion of repolarization [24]. AP alternans, defined as changes in AP occurring on an every-other-beat basis, can be a basis for TWA. Ischemia, extrasystoles or a sudden HR change may cause discordant (spatially unsynchronized) alternans and unidirectional block, thus setting the stage for ventricular arrhythmias like VF [24].

Ventricular dispersion properties at different HRs are usually quantified by the so-called dynamic APD restitution curves (APDR). These curves, see Fig. 13 left panel, express the APD as a function of the RR interval (inverse of HR) for different regions within the myocardium. In experimental, clinical and computational studies, it has been shown that an increase in APDR dispersion is associated with greater propensity to suffer from VT/VF, the most common sequence to SCD [25], [26]. Other studies have reported differences in transmural heterogeneity at different cycle lengths between end-stage failing and non-failing human ventricles [27]. When compared to non-failing hearts, end-stage failing hearts presented significantly decreased transmural APD gradients between the subendocardium and subepicardium. All these evidences highlight the challenge of identifying surface ECG surrogates of this APDR dispersion present at cellular and tissue levels. Some of those will be reviewed in sections III-D2 and III-D3, together with their capacity for arrhythmia prediction.

Cardiac arrhythmias can be caused by abnormalities in impulse formation, impulse conduction, or both, as further detailed in the following section.

\section{2) Abnormalities of impulse generation:}

a) Automaticity: Abnormal automaticity occurs when cells other than those in the sino-atrial (SA) node undertake its function. Certain forms of VT arise due to such abnormalities. Under pathological conditions, the SA node cells may reduce their rate of spontaneous depolarization or even lose their property of automaticity.

b) Afterdepolarizations and triggered activity: If, under normal SA node functioning, other cells develop rates of firing faster than those of the SA node, new APs are initiated in those cells and their adjacent ones. This triggered activity can be the 
result of the formation of early afterdepolarizations (EADs, second depolarizations occurring during AP repolarization) or delayed afterdepolarizations (DADs, occurring after AP repolarization). A good number of investigations have pointed to EADs playing a role in the initiation and perpetuation of the polymorphic VT known as Torsades de Pointes (TdP) [28].

3) Abnormalities of impulse conduction: Abnormal impulse conduction may lead to reentry, where a circuitous wavefront reexcites the same tissue indefinitely. Unidirectional conduction block and slow conduction are required for reentry to occur. VF is an example of re-entrant arrhythmia.

\section{Biophysical modeling of the ECG}

In this section we describe a modeling approach that considers the myocardium as a volume conductor with two surfaces uniformly bounding the whole ventricular tissue, also known as Uniform Double Layer (UDL) [29], giving raise to the Dominant $\mathrm{T}$ wave concept [12]. This is derived from an analysis of the electrical properties of the ventricle treated as a homogeneous syncytium by means of the bidomain approach [30]. This approach assumes that the myocardial tissue is formed by two separate domains, the intracellular and the extracellular spaces, sharing the same volume [31]. Both domains behave as regular volume conductors and, therefore, two potentials are defined at each point.

The bidomain model is commonly employed in large-scale simulations with different applications. Here our interest is in obtaining the potential recorded at the body surface [30]. This results in an inhomogeneous volume conductor problem constituted by the torso with the ventricular cavities. In the frequency range of interest $(\leq 1000 \mathrm{~Hz})$, the potential $x(t)$ recorded at a given unipolar ECG can be written as

$$
x(t)=-\int_{\mathcal{H}} c_{i}\left(\nabla_{v} \phi_{m}(\mathbf{v}, t) \cdot \nabla_{v} Z(\mathbf{v})\right) d \mathbf{v}
$$

where $\phi_{m}(\mathbf{v}, t)$ is the transmembrane potential (TMP) (difference of potential between the inside and the outside of the cell), $c_{i}$ is the inner domain conductance tensor, and $\nabla_{v} Z(\mathbf{v})$ is the transfer impedance function, which relates current dipole $c_{i} \boldsymbol{\nabla}_{v} \phi_{m}(\mathbf{v}, t)$ in the volume $d \mathbf{v}$ with its contribution to the potential in the unipolar lead. These contributions are integrated over the heart volume $\mathcal{H}$, coordinated by $\mathbf{v}$. When both domains have the same anisotropy ratio, equation (1) is equivalent [32] to the surface integral

$$
x(t)=-\int_{\mathcal{S}} c_{i} \phi_{m}(\mathbf{s}, t)\left(\nabla_{v} Z(\mathbf{s}) \cdot d \overrightarrow{\mathbf{s}}\right)
$$

where $\mathcal{S}$ is the surface, coordinated by $s$, enclosing the active regions of the heart (endocardium, epicardium and septum). Although the cardiac tissue does not satisfy well the condition of equal anisotropy, it has been shown for 2D cardiac tissue [33] that the approximation essentially holds, except in the neighborhood of the activation site.

According to (2), the potential $x(t)$ can be obtained by integrating only over the surface $\mathcal{S}$. Therefore, we can replace the active sources in the heart by a dipole layer on $\mathcal{S}$, with a moment proportional to $\phi_{m}(t)$ without affecting $x(t)$. This equivalence, linking the potential measured in a lead with the
TMP at the surface $\mathcal{S}$ is usually called (equivalent) surface source model.

Dominant $T$ wave formalism: In [29], van Oosterom pointed out that using equation (2) to obtain body surface $\mathrm{T}$ waves from the electrical activity in the heart is equivalent to evaluate a linear system for each time. The surface of the heart can be divided into $M$ contiguous regions (called nodes), where each node is treated as a single lumped source. Considering $L$ surface leads, equation (2) can be approximated, at any instant $t$, by

$$
\left[x_{1}(t), \ldots, x_{L}(t)\right]^{T}=\mathbf{x}(t)=\mathbf{A}\left[\phi_{1}(t), \ldots \phi_{M}(t)\right]^{T}
$$

where $\mathbf{x}(t)$ is a column vector with the $L$ potentials, and A is an $L \times M$ transfer matrix, invariable for a given lead configuration and patient, and accounting for the geometry and conductivity of the volume conductor, as well as for the solid angles under which each node contributes to the potentials in $\mathbf{x}(t)$. In the rest of this work, we will use $\phi_{m}(t)$ to describe the repolarization phase of the equivalent TMP of a given region $m$. Note that the sum of the $M$ elements of each row of matrix A must be zero (i.e. $\mathbf{A e}_{1}=\mathbf{0}$, where $\mathbf{e}_{1}$ and is an $M \times 1$ vector of ones and $\mathbf{0}$ is a $L \times 1$ vector of zeros). This property shows that a $\mathrm{T}$ wave in the surface ECG is only possible if $\phi_{m}(t)$ differs between regions. As stated in [29], eq. (3) allows to link the shape of the $\mathrm{T}$ wave in each lead to the different TMPs. If we further assume that the different $\phi_{m}(t)$ have the same shape and only differ in the time of repolarization time $(\mathcal{R T}) \rho_{m}$, i.e., $\phi_{m}(t)=d\left(t-\rho_{m}\right)$, where $\rho_{m}$ is defined as the time with maximum downslope of the TMP $d(t)$, then, as proposed in [34],

$$
\mathbf{x}(t)=\mathbf{A}\left[d\left(t-\rho_{1}\right), \ldots, d\left(t-\rho_{M}\right)\right]^{T} .
$$

This approximation essentially assumes that the TMP downslope shape is approximately constant across the heart surface. Expressing the $\mathcal{R} \mathcal{T}$ of each node as [34]

$$
\rho_{m}=\bar{\rho}+\Delta \rho_{m},
$$

where $\bar{\rho}_{=} \sum_{m=1}^{M} \rho_{m} / M$, when $\Delta \rho_{m} \ll \bar{\rho}$ the TMP shape $d(t)$ can be expanded in series around $\bar{\rho}$ as

$$
\begin{aligned}
d\left(t-\rho_{m}\right) & =d(t-\bar{\rho})-\left.\Delta \rho_{m} \frac{d d(\tau)}{d \tau}\right|_{\tau=t-\bar{\rho}} \\
+ & \left.\frac{\Delta \rho_{m}^{2}}{2 !} \frac{d^{2} d(\tau)}{d \tau^{2}}\right|_{\tau=t-\bar{\rho}}+o\left(\Delta \rho_{m}^{3}\right) .
\end{aligned}
$$

Since $\mathbf{A e}_{1} D(t-\bar{\rho})=0$ and neglecting higher order terms, the model (4) can be approximated as

$$
\mathbf{x}(t) \approx-\mathbf{A} \boldsymbol{\Delta} \boldsymbol{\rho} \dot{d}(t-\bar{\rho})
$$

with $\boldsymbol{\Delta} \boldsymbol{\rho}=\left[\Delta \rho_{1}, \Delta \rho_{2}, \ldots, \Delta \rho_{M}\right]^{T}$, or in discrete time

$$
\mathbf{X} \approx \mathbf{w}_{1} \mathbf{t}_{d}^{T}
$$

where $\mathbf{w}_{1}=-\mathbf{A} \Delta \boldsymbol{\rho}$ is an $L \times 1$ vector of the so-called lead factors, $\mathbf{X}$ is an $L \times N$ matrix with the sampled signals at the surface leads and the $N \times 1$ vector $\mathbf{t}_{d}$ is a sampled version of $t_{d}(t)=\dot{d}(t-\bar{\rho})$. The vector $-\mathbf{t}_{d}$ was given the name of dominant $T$ wave by van Oosterom [35]. Note that if the approximation in (7) holds, all $\mathrm{T}$ waves measured on different 
leads are just a scaled version of $\mathbf{t}_{d}$. Methods to estimate $\mathbf{t}_{d}$ and $\mathbf{w}_{1}$ can be found in [34], [35].

This approximated modeling to derive the $\mathrm{T}$ wave can be adapted to situations with increased dispersion of the $\mathcal{R} \mathcal{T}_{\mathrm{s}}$, as it happens in patients with increased vulnerability to ventricular arrhythmias [36]. In that case, the second order contribution in (6) becomes relevant and the following secondorder approximation of (4) can be used:

$$
\begin{gathered}
\mathbf{x}(t) \approx-\mathbf{A} \boldsymbol{\Delta} \boldsymbol{\rho} \dot{d}(t-\bar{\rho})+\frac{1}{2} \mathbf{A} \boldsymbol{\Delta} \boldsymbol{\rho}^{2} \ddot{d}(t-\bar{\rho}) \\
\mathbf{X} \approx \mathbf{w}_{1} \mathbf{t}_{d}^{T}+\mathbf{w}_{2} \dot{\mathbf{t}}_{d}^{T}
\end{gathered}
$$

where $\mathbf{w}_{2}=\frac{1}{2} \mathbf{A} \boldsymbol{\Delta} \boldsymbol{\rho}^{2}$ is a set of second-order lead factors and $\boldsymbol{\Delta} \boldsymbol{\rho}^{2}=\left[\Delta \rho_{1}^{2}, \Delta \rho_{2}^{2}, \ldots, \Delta \rho_{M}^{2}\right]^{T}$. One example of the $d\left(t-\rho_{m}\right), m=1, \ldots, M$ and the T waves generated with the methodology is depicted in Fig. 6. For this model to be used, we need to estimate both $\mathbf{t}_{d}$ and the lead vectors $\mathbf{w}_{1}$ and $\mathbf{w}_{2}$, from the original data $\mathbf{X}$.

Lead factor estimation: One simple option to estimate the dominant $\mathrm{T}$ wave $\mathbf{t}_{d}$ is as the average of all the $\mathrm{T}$ waves weighted by their integral [35],

$$
\mathbf{t}_{d}^{T}=c_{1} \mathbf{e}_{1}^{T} \mathbf{X}^{T} \mathbf{X}
$$

and multiplying equation (8) by $\mathbf{e}_{1}$ we obtain

$$
\mathbf{w}_{1}=\frac{\mathbf{X} \mathbf{e}_{1}}{\mathbf{t}_{d}^{T} \mathbf{e}_{1}}
$$

which is a close expression for the first order approximation lead factor $\mathbf{w}_{1}$. The scalar $c_{1}(11)$ is defined as in [34]. Another alternative is to estimate the dominant $\mathrm{T}$ wave as the first principal component (PCA) of the $\mathrm{T}$ waves by doing a PCA decomposition in time [37] of the $\mathrm{T}$ wave matrix $\mathbf{X}$. This can be done equivalently by singular value decomposition (SVD) [38], [39]:

$$
\mathbf{X}=\mathbf{U} \boldsymbol{\Lambda} \mathbf{V}^{T}=\sum_{l=1}^{L} \mathbf{u}_{l} \lambda_{l} \mathbf{v}_{l}^{T}
$$

resulting in

$$
\mathbf{t}_{d}^{T}=c_{2} \lambda_{1} \mathbf{v}_{1}^{T}, \quad \mathbf{w}_{1}=\mathbf{u}_{1} / c_{2}
$$

which if $\lambda_{1} \gg \lambda_{l \neq 1}$ can be proved [34] to be equivalent to $\mathrm{T}$ wave averages. $c_{2}$ is defined as in [34]. This SVDbased estimate can be shown to be optimum in the sense of minimizing the Frobenius norm $\epsilon_{1}=\left\|\mathbf{X}-\mathbf{w}_{1} \mathbf{t}_{d}^{T}\right\|_{F}$. The second order approximation can be done as in [34] by minimization of the norm $\epsilon_{2}=\left\|\mathbf{X}-\mathbf{w}_{1} \mathbf{t}_{d}^{T}-\mathbf{w}_{2} \dot{\mathbf{t}}_{d}^{T}\right\|_{F}$. However, other alternatives exist by realizing that minimizing $\epsilon_{2}$ reduces to minimizing $\epsilon_{1}$ by considering now $\left(\mathbf{X}-\mathbf{w}_{1} \mathbf{t}_{d}^{T}\right)$ as the $\mathbf{X}$ in $\epsilon_{1}$ and $\mathbf{w}_{2} \dot{\mathbf{t}}_{d}^{T}$ as the $\mathbf{w}_{1} \mathbf{t}_{d}^{T}$. In such a case $\dot{\mathbf{t}}_{d}^{T}$ becomes proportional to the first eigenvalue of $\left(\mathbf{X}-\mathbf{w}_{1} \mathbf{t}_{d}^{T}\right)$, which since $\mathbf{w}_{1} \mathbf{t}_{d}^{T}$ is already the first component of $\mathbf{X}$ then it becomes evident that $\dot{\mathbf{t}}_{d}^{T}$ can be estimated by the second eigenvector of $\mathbf{X}$ as:

$$
\dot{\mathbf{t}}_{d}^{T}=c_{3} \lambda_{2} \mathbf{v}_{2}^{T}, \quad \mathbf{w}_{2}=\mathbf{u}_{2} / c_{3}
$$

where $c_{2}$ and $c_{3}$ are just proportionality factors interchangeable between the dominant $\mathrm{T}$ wave and lead factors [34]. For later use in section III-B2, we can note that equation (10) now becomes

$$
\mathbf{X} \approx \lambda_{1} \mathbf{u}_{1} \mathbf{v}_{1}^{T}+\lambda_{2} \mathbf{u}_{2} \mathbf{v}_{2}^{T}
$$

\section{ECG REPOLARIZATION RISK MARKERS}

\section{A. ECG processing for repolarization analysis}

Prior to computation of ECG repolarization indices, the following four processing steps are commonly applied:

1) ECG filtering and preconditioning: This includes removal of muscle noise, powerline interference and baseline wander [3]. The ECG signal recorded in lead $l$ is denoted by $x_{l}(n)$ after filtering, while for the multi-lead filtered signal the vector $\mathbf{x}(n)=\left[x_{1}(n) \ldots x_{L}(n)\right]^{T}$ is used.

2) QRS detection: Beat detection provides a series of samples $n_{i}$ and its related RR intervals $R R_{i}=n_{i}-n_{i-1}, i=$ $0 \ldots B$, corresponding to the detected QRS complexes.

3) Wave delineation: Automatic determination of wave boundaries and peaks is performed (see Fig. 3). The most relevant points for repolarization analysis are the QRS boundaries, $\mathrm{T}$ wave boundaries and $\mathrm{T}$ wave peak. Commonly computed repolarization intervals, evaluated for each beat $i$, are the QT interval $\left(Q T_{i}\right.$, between QRS onset and T wave end), RT interval $\left(R T_{i}\right.$, between QRS fiducial point and $\mathrm{T}$ wave peak), $\mathrm{T}$ wave width $\left(T_{\mathrm{w}_{i}}\right)$ and $\mathrm{T}$ wave peak-to-end $\left(T_{\mathrm{pe}_{i}}\right)$.

Different delineation approaches have been proposed in the literature. Multiscale analysis based on the dyadic wavelet transform, allowing representation of a signal's temporal features at different resolutions, has proved useful for QRS detection and ECG delineation [41]. Multi-lead delineation, either based on selection rules applied to single-lead delineation results or based on VCG processing, has shown improved accuracy and stability [42].

4) Segmentation: A repolarization segmentation window $W_{i}$, usually containing the ST-T complex, can be defined. The beginning of the window can be set at fixed or RR-dependent offsets from the QRS fiducial point or the QRS end. An alignment stage can be applied if synchronization is required. If an $N$-sample window, $W_{i}$, beginning at sample $n_{i}^{\mathrm{w}}$ is defined for each $i$ th beat to contain its repolarization phase, the extracted repolarization segment for the $i$ th beat and $l$ th lead can be denoted as $x_{i, l}(n)=x_{l}\left(n_{i}^{\mathrm{w}}+n\right), n=0, \ldots, N-1$. For multilead analysis, the $L \times 1$ vector $\mathbf{x}_{i}(n)=\left[x_{i, 1}(n), \ldots, x_{i, L}(n)\right]^{T}$ contains samples in the different leads.

\section{B. ECG markers of spatial repolarization dispersion}

In this section a review of ECG indices proposed in the literature to assess spatial heterogeneity of ventricular repolarization is presented.

1) Dispersion of repolarization reflected on ECG intervals: QT dispersion $\left(Q T_{d}\right)$, computed as the difference between the maximum and minimum QT values across leads, was proposed to quantify ventricular repolarization dispersion (VRD) [43]. However, the relationship between $Q T_{d}$ and VRD resulted controversial [44], as has been shown to mainly reflect the different lead projections of the $\mathrm{T}$ wave loop rather than any other type of dispersion. As a result, $Q T_{d}$ has not been further considered as a VRD index. 

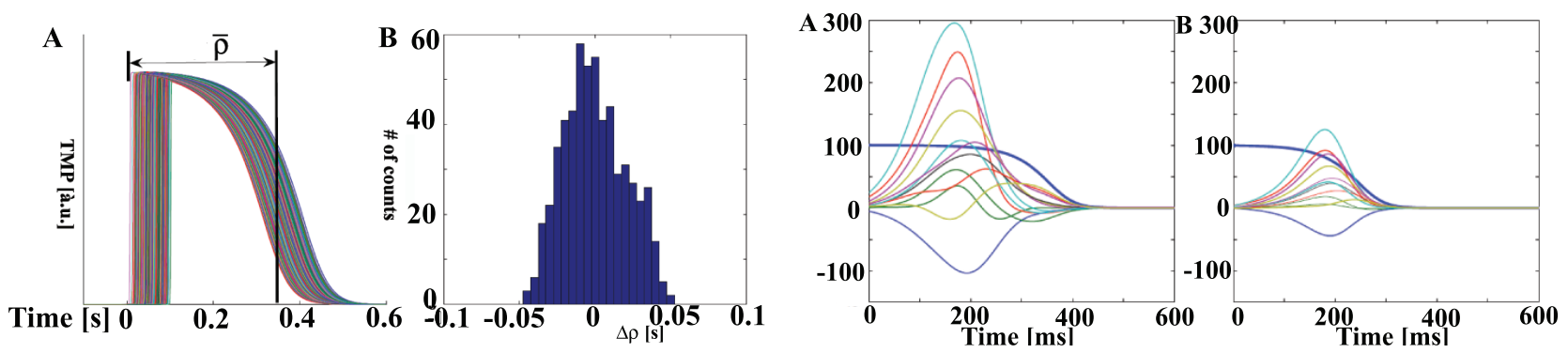

Fig. 6. Superposition of transmural potentials $d\left(t-\rho_{m}\right)$ of each node (left A), the histogram of $\Delta \boldsymbol{\rho}$ (left B) and the generated T waves for a large/low range of $\mathcal{R} \mathcal{T}, \rho_{m}$ (right A/B). Reproduced from [40].

In [45] isolated-perfused canine hearts were used to measu $Q T_{d}$ and T wave width, $T_{\mathrm{w}}$. VRD values were computed aft changing temperature, cycle length and activation sequenc VRD, evaluated directly from recovery times of epicardi potentials, was compared to $T_{\mathrm{w}}$ and $Q T_{d}$ and shown to strongly correlated with $T_{\mathrm{w}}$, but not with $Q T_{d} . T_{\mathrm{w}}$ was al confirmed as a VRD measurement in a rabbit heart moc where increased dispersion was generated by d-sotalol a] premature stimulation [46]. $T_{\mathrm{w}}$ is a complete measure dispersion as evidenced on the ECG. When addressing the problem of evaluating $T_{\mathrm{w}}$ in recordings under ischemia [47], which largely increases repolarization dispersion, the $\mathrm{T}$ onset estimation can largely be affected by the ST elevation, making $T_{\mathrm{w}}$ estimation unreliable and then being $T_{p e}$ a possibly better option. Even if $T_{p e}$ does not only reflect transmural dispersion but may include also other ventricular heterogeneities (e.g. apico-basal) [48], [49], it is still a marker of VRD that can be quantified from the ECG.

Since ECG wave onsets and ends have interlead variability, due to the different projections of the cardiac electrical activity, and also individual lead measures are more easily affected by noise, multi-lead criteria are some times preferred [50]. In this way the estimated interval value includes electrical activity recovered at the complete space represented by the lead set. $\mathrm{T}$ wave onset can be measured as the earliest reliable $\mathrm{T}$ wave onset across leads and $\mathrm{T}$ wave end as the latest reliable $\mathrm{T}$ wave end across leads, obtained either by applying rules, as proposed in [50] to quantify $T_{\mathrm{w}}$, or by VCG-based methods [51].

2) Dispersion of repolarization reflected on $T$ wave morphology: Several indices have been proposed to describe the $\mathrm{T}$ wave shape. They lie on the assumption that larger dispersion in repolarization times results in a more complex $\mathrm{T}$ wave shape. Some of these descriptors rely on PCA to extract information from the $\mathrm{T}$ wave shape [52]. The total cosine R-to-T, $T_{\mathrm{CRT}}$, is defined as the cosine of the angle between the dominant vectors of depolarization and repolarization phases in a 3D loop and has been evaluated to compare repolarization in healthy subjects and hypertrophic cardiomyopathy patients [53]. If the original ECG has $L$ leads $\left(\mathbf{x}_{i}(n)\right.$ in vector notation), it is transformed to $\boldsymbol{\omega}_{i}(n)=$ $\left[\begin{array}{llll}\omega_{i, 1}(n) & \omega_{i, 2}(n) & \ldots & \omega_{i, L}(n)\end{array}\right]^{T}$, as

$$
\boldsymbol{\omega}_{i}(n)=\mathbf{U}_{i}^{T} \mathbf{x}_{i}(n),
$$
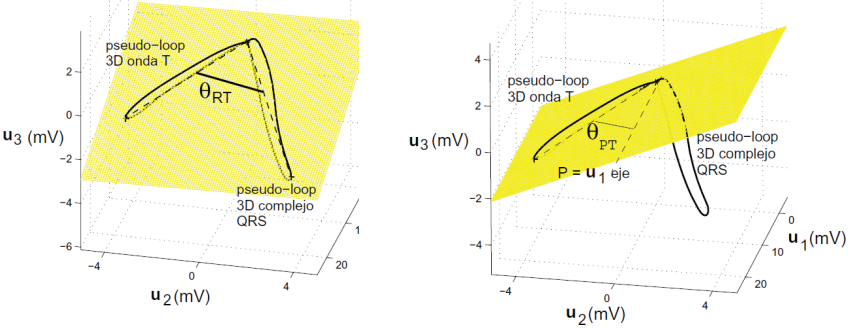

Fig. 7. R-to-T Angle (left) between repolarization and depolarization phases. The Principal component-to-T angle (right) between a fix reference and the repolarization, Adapted from [46].

where $\mathbf{U}_{i}$ is the $[L \times L]$ matrix whose columns are the eigenvectors of the $i$-th beat interlead autocorrelation matrix (computed in the whole PQRST complex). Then, a 30-ms window ( $N_{\text {QRS }}$ samples) is defined centered on the QRS fiducial point $n_{i}$. The $\mathrm{T}$ wave peak position, $n_{i, \mathrm{~T}}$, is estimated as the position in the ST-T complex with maximum $\left|\boldsymbol{\omega}_{i}(n)\right|$. Then, the index $T_{\mathrm{CRT}_{i}}$ is defined by

$$
T_{\mathrm{CRT}_{i}}=\frac{1}{N_{\mathrm{QRS}}} \sum_{n=0}^{N_{\mathrm{QRS}}-1} \cos \angle\left(\boldsymbol{\omega}_{i}(n), \boldsymbol{\omega}_{i}\left(n_{i, \mathrm{~T}}\right)\right) .
$$

If only tracking of the ventricular transmural gradient in the same recording is needed, it is possible to estimate the gradient of repolarization with respect to a fixed reference, assuming that the direction of depolarization does not change with repolarization heterogeneity. The proposed index is called Total angle principal component-to- $T, T_{\mathrm{PT}}$, [46] see Fig.7. The reference $\mathbf{u}$ can be taken to be the unitary vector in the first principal component direction, yielding

$$
T_{\mathrm{PT}_{i}}=\angle\left(\mathbf{u}, \boldsymbol{\omega}_{i, \mathrm{D}}\left(n_{i, \mathrm{~T}}\right)\right) .
$$

Total morphology dispersion, $T_{\mathrm{MD}}$, is an index computed by selecting the first three principal components of the ECG (assumed to be the dipolar components) and reconstructing the signal in the original leads after discarding the rest of components. Splitting the eigenvectr matrix as $\mathbf{U}_{i}=$ $\left[\begin{array}{ll}\mathbf{U}_{i, 3} & \mathbf{U}_{i, L-3}\end{array}\right]$ and applying

$$
\hat{\mathbf{x}}_{i}(n)=\mathbf{U}_{i, 3} \mathbf{U}_{i, 3}^{T} \mathbf{x}_{i}(n),
$$

the "dipolar" signal $\hat{\mathbf{x}}_{i}(n)$ is obtained. This signal is again processed by SVD, but now defined only from the spatial correlation of the ST-T complex, obtaining the transformation matrix $\check{\mathbf{U}}_{i}$. Now the matrix is truncated to its first two columns $\check{\mathbf{U}}_{i, 2}$ 
(defining the main plane of variation of the repolarization) and again a signal is reconstructed in the original lead set: $\check{\mathbf{x}}_{i}(n)=$ $\check{\mathbf{U}}_{i, 2} \check{\mathbf{U}}_{i, 2}^{T} \hat{\mathbf{x}}_{i}(n)$. Note that $\check{\mathbf{U}}_{i, 2}=\left[\begin{array}{lll}\boldsymbol{\phi}_{i, 1} & \cdots & \boldsymbol{\phi}_{i, L}\end{array}\right]^{T}$, where $\phi_{i, l}$ are $2 \times 1$ reconstruction vectors, which can be seen as the direction into which the SVD-transformed signal has to be projected to get each original lead in $\check{\mathbf{x}}_{i}(n)$. For each pair of leads $l_{1}$ and $l_{2}$ the angle between both directions is

$$
\alpha_{l_{1}, l_{2}}(i)=\angle\left(\phi_{i, l_{1}}, \phi_{i, l_{2}}\right) \in\left[0^{o}, 180^{\circ}\right],
$$

measuring the morphology difference between leads $l_{1}$ and $l_{2}$ (a small angle is associated with similar shape in both leads). The non-normalized $T_{\mathrm{MD}_{i}}$ index is computed by averaging these angles for all pairs of leads,

$$
T_{\mathrm{MD}_{i}}=\frac{1}{L(L-1)} \sum_{\substack{l_{1}, l_{2}=1 \\ l_{1} \neq l_{2}}}^{L} \alpha_{l_{1}, l_{2}}(i)
$$

reflecting the average repolarization morphology dispersion between leads. In the original definition of $T_{\mathrm{MD}}$ each $\phi_{i, l}$ was multiplied by its corresponding eigenvalue, having a different geometrical interpretation [53].

Other descriptors have been proposed, based on the distribution of the eigenvalues of the inter-lead repolarization correlation matrix $\hat{\mathbf{R}}_{x_{i}}=\sum_{n=0}^{N-1} \mathbf{x}_{i}(n) \mathbf{x}_{i}^{T}(n)$. Let us denote the eigenvalues as $\lambda_{i, j}, j=1 \ldots L$, sorted in descending order. The energy of the dipolar components is given by the sum of three first eigenvalues, while the sum of the rest of eigenvalues represents the energy of the non-dipolar components. The $T$ wave residuum, $T_{\mathrm{wR}}$ is defined as

$$
T_{\mathrm{WR}_{i}}=\sum_{j=4}^{L} \lambda_{i, j} / \sum_{j=1}^{L} \lambda_{i, j}
$$

and can be interpreted as the relative energy of the non-dipolar components [44], [53]. This is based on the hypothesis that in normal conditions, the ECG can be explained by the first three components (dipolar components). When local repolarization heterogeneities are present, the dipolar model does not hold any longer and this is reflected in larger eigenvalues corresponding to the non-dipolar components, thus increasing $T_{\mathrm{WR}_{i}}$ values.

$T$ Wave Uniformity, $T_{u}$, and $T$ wave Complexity, $T_{c}$, defined as

$$
T_{u_{i}}=\lambda_{i, 1} / \sum_{j=1}^{L} \lambda_{i, j} \quad, \quad T_{c_{i}}=\sum_{j=2}^{L} \lambda_{i, j} / \sum_{j=1}^{L} \lambda_{i, j}=1-T_{u_{i}},
$$

are two other indices based on the same approach, aiming to quantify the morphology of the ST-T complex loop [52]. A $T_{u}$ value close to one indicates that the ST-T complex loop is very narrow and lies most of the time in the direction defined by the first eigenvector of the SVD decomposition. On the other hand, $T_{c}$ close to one means that the loop is mainly contained in a plane. The $\mathrm{T}$ wave complexity has also been alternatively defined as the second to first eigenvalue ratio,

$$
T_{c_{i}}^{\prime}=\lambda_{i, 2} / \lambda_{i, 1}
$$

which in the framework of this review can be justified in the light of equations (16) and (9): the larger $\lambda_{2}$ is with respect to $\lambda_{1}$ (larger $T_{c}^{\prime}$ ), the larger is the second order term in the approximation (9) and, thus, the larger the $\mathcal{R} \mathcal{T}$ dispersion $\Delta \boldsymbol{\rho}$, therefore providing extra support for this measure as a VRD index and illustrating an example of physiologicallydriven method development. The geometrical interpretation of $T_{c}^{\prime}$ refers to the roundness of the loop (also denoted in some works as $\left.T_{2,1}\right)$. It has been shown that $T_{c}^{\prime}$ is higher in patients with LQTS than in healthy subjects [52]. Also the nonplanarity of the ventricular repolarization can be measured as $T_{3,1_{i}}=\lambda_{i, 3} / \lambda_{i, 1}[47]$.

Finally, some $\mathrm{T}$ wave shape indices such as $\mathrm{T}$ wave amplitude $\left(T_{\mathrm{A}}\right)$, the ratio of the areas at both sides of the $\mathrm{T}$ peak $\left(T_{\mathrm{RA}}\right)$ and the ratio of the $\mathrm{T}$ peak to boundary intervals at both sides of the $\mathrm{T}$ peak $\left(T_{\mathrm{RT}}\right)$ (Fig. 3) have been proposed as risk markers [54], grounded on the evidence that increased VRD resulted in taller and more symmetric $\mathrm{T}$ waves [55].

The value of these makers to characterize VRD during the first minutes of acute ischemia induced by percutaneous coronary intervention (PCI) has also been studied [47]. It was observed that changes in PCA-based morphology descriptors were very dependent on the occluded artery, suggesting that morphology changes are very affected by the direction of the equivalent injury current. Most of the studied indices presented a large inter-individual variability, pointing to the necessity of using patient-adapted indices of relative changes.

\section{ECG markers of temporal repolarization dispersion}

ECG indices proposed in the literature as markers of temporal heterogeneity of ventricular repolarization are reviewed in this section together with their links to ventricular arrhythmias. The meaning of temporal is taken as it goes beyond a single beat and includes information present in the evolution of the index across several beats.

1) QT adaptation to HR changes: The QT interval is to a great extent influenced by changes in HR [56]. A variety of HR-correction formulas have been proposed in the literature to compare QT measurements at different HRs [57]. Prolongation of the QT interval or of the corrected QT interval $\left(Q T_{c}\right)$ have been recognized in some studies as markers of arrhythmic risk [58]. However, it is today widely acknowledged that QT or $Q T_{c}$ prolongation per se are poor surrogates for proarrhythmia [28]. The most popular formula to correct the QT interval for the effects of HR is Bazett's formula $\left(Q T_{c}=Q T / \sqrt{R R}\right)$, but evidences of large overcorrection at low HR and undercorrection at high HR have led to other formulas such as the Fridericia formula $\left(Q T_{c}=Q T / R R^{1 / 3}\right)$, with better clinical acceptance today.

Importantly, under conditions of unstable HR, the QT hysteresis lag after HR changes needs to be taken into consideration. The QT interval requires some time to reach a new steady state following a HR change, with important information for prediction of arrhythmias additionally found in this adaptation time [59]. In the literature, QT hysteresis has been evaluated under various conditions. In [60] the ventricular paced QT interval was shown to take between 2 and 3 minutes to follow 
a change in HR, with the adaptation process presenting two phases: a fast initial phase lasting for a few tens of seconds and a second slow phase lasting for several minutes. In [59], QT adaptation was analyzed after a provoked HR change or after physical exercise and the QT hysteresis lag was of some minutes.

The ionic mechanisms underlying QT interval rate adaptation have been investigated with the techniques described in section II-A and II-C [61]. The time for 90\% QT adaptation in simulations was of $3.5 \mathrm{~min}$, in agreement with experimental and clinical data in humans, see Fig. 8. APD adaptation was shown to follow similar dynamics to QT interval, being faster in midmyocardial cells $(2.5 \mathrm{~min})$ than in endocardial and epicardial cells ( $3.5 \mathrm{~min}$ ), with these times being in accordance with experimental data in human and canine tissues [61], [62]. Both QT and APD adapt in two phases: a fast initial phase with time constant of around $30 \mathrm{~s}$, mainly related to the Ltype calcium and the slow delayed rectifier potassium current, and a second slow phase of 2 min driven by intracellular sodium concentration $\left([\mathrm{Na}]_{i}\right)$ dynamics. The investigations in [61] support the fact that protracted QT adaptation can provide information of increased risk for cardiac arrhythmias.
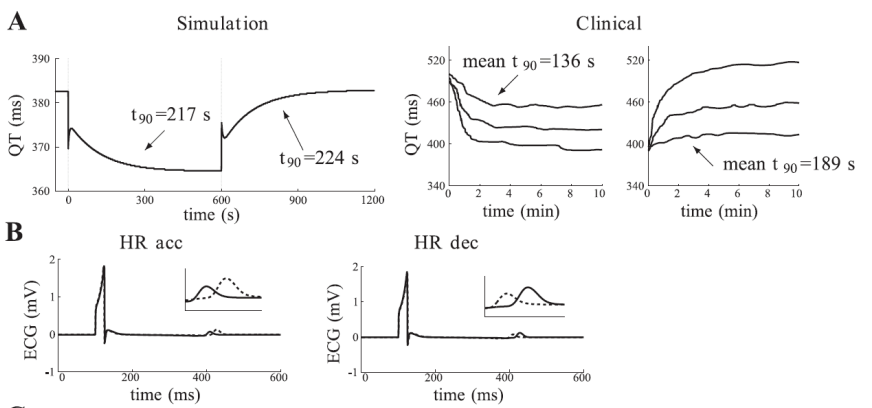
HR dec

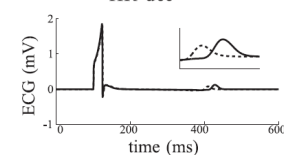

$\mathrm{C}$

\begin{tabular}{|c|c|c|c|}
\hline Simulation & TP06 & HRacc: $217 \mathrm{~s}$ & HRdec: $224 \mathrm{~s}$ \\
\hline \multirow{4}{*}{ Clinical } & Lau et al & HRacc, mean \pm SEM: $136 \pm 16 \mathrm{~s}$ & HRdec, mean \pm SEM: $189 \pm 25 \mathrm{~s}$ \\
\cline { 2 - 4 } & Seed et al & $>180 \mathrm{~s}$ \\
\cline { 2 - 3 } & Attwell et al & $\sim 180 \mathrm{~s}$ \\
\cline { 2 - 3 } & Grom et al & $60 \mathrm{~s}-300 \mathrm{~s}$ \\
\cline { 2 - 3 } & Pueyo et al & $10 \mathrm{~s}-240 \mathrm{~s}$ \\
\hline
\end{tabular}

Fig. 8. A: left: simulated QT interval adaptation in human pseudo-ECG for cycle length (RR) changes from 1000 to $600 \mathrm{~ms}$ and latter back to $1000 \mathrm{~ms}$; right: QT adaptation in human ECG recordings from 50 or 110 beats $/ \mathrm{min}$ in increments or decrements of 20, 40, and 60 beats/min. Time required for $90 \%$ QT rate adaptation $\left(t_{90}\right)$ is presented. B: simulated pseudo-ECGs corresponding to first (dotted line) and last (solid line) beats after RR decrease (left) and RR increase (right). C: $t_{90}$ values for simulated pseudo-ECGs and clinical human ECGs. HR Acc, acceleration; Dec, deceleration; TP06, human ventricular cell model developed in [14]. Reproduced from [61].

In view of previous findings, it is well motivated to introduce a method to assess and quantify QT adaptation to spontaneous HR changes in Holter ECGs, in [63] applied to recordings of post-myocardial infarction (MI) patients. The method investigates QT dependence on HR by building weighted averages of RR intervals preceding each QT measurement. The relationship between the QT interval and the RR interval is specifically modeled using a system composed of a FIR filter followed by a nonlinear biparametric regression function (see Fig. 9). The input to the system is defined from the resampled beat-to-beat $R R_{i}$ interval series (denoted by $x_{\mathrm{RR}}(k)$, where $k$ is discrete time), the output is the resampled $Q T_{i}$ interval series $\left(y_{\mathrm{QT}}(k)\right)$, and additive noise $v(k)$ is considered so as to include e.g. delineation and modeling errors. The first (linear) subsystem describes the influence of previous RR intervals on each QT measurement, while the second (nonlinear) subsystem is representative of how the QT interval evolves as a function of the weighted average RR measurement, $\overline{R R}$, obtained at the output of the first subsystem.

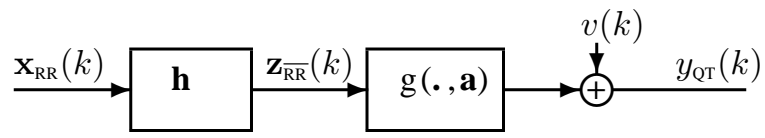

Fig. 9. Block diagram describing the $[R R, Q T]$ or or $\left[R R, T_{p e}\right]$ relationship consisting of a time invariant FIR filter (impulse response $\mathbf{h}$ ) and a nonlinear function $g_{k}(., \mathbf{a})$ described by the parameter vector $\mathbf{a} . v(k)$ accounts for the output error. Reproduced from [63].

The global input-output relationship is thus expressed as:

$$
y_{\mathrm{QT}}(k)=g\left(\mathbf{z}_{\overline{\mathrm{RR}}}(k), \mathbf{a}\right)+v(k),
$$

where

$$
\mathbf{z}_{\overline{\mathrm{RR}}}(k)=\left[\begin{array}{ll}
1 & z_{\overline{\mathrm{RR}}}(k)
\end{array}\right]^{T}=\left[\begin{array}{ll}
1 & \mathbf{h}^{T} \mathbf{X}_{\mathrm{RR}}(k)
\end{array}\right]^{T} .
$$

In the above expressions,

$$
\mathbf{x}_{\mathrm{RR}}(k)=\left[\begin{array}{llll}
x_{\mathrm{RR}}(k) & x_{\mathrm{RR}}(k-1) & \ldots & x_{\mathrm{RR}}(k-N+1)
\end{array}\right]^{T},
$$

is the history of $R R$ intervals, $\mathbf{h}=\left[\begin{array}{lll}h_{0} & \ldots & h_{N-1}\end{array}\right]^{T}$ is the impulse response of the FIR filter and $g(\cdot)$ is the regression function parameterized by vector $\mathbf{a}=\left[\begin{array}{ll}a_{0} & a_{1}\end{array}\right]^{T}$ (see [63] for a list of used regression functions). Identification of the unknown system is performed individually for each patient using a global optimization algorithm. According to the results in [63], the QT interval requires nearly 2.5 minutes to follow HR changes, in mean over patients, although both the duration and profile of QT hysteresis are found to be highly individual. As previously commented for the results reported in [61], the adaptation process is shown to be composed of two distinct phases: fast and slow.

The methodology described in [63] has been subsequently extended in [64] to describe temporal changes in QT dependence on HR, i.e. to account for possibly different adaptation characteristics along each recording. The linear and nonlinear subsystems used to model the $Q T / R R$ relationship are then considered to be time-variant. An adaptive approach based on the Kalman Filter is used in [64] to concurrently estimate the system parameters. It has been shown that QT hysteresis can range from a few seconds to several minutes depending on the magnitude of HR changes along a recording.

The clinical value of investigating QT interval adaptation to HR as a way to provide information on the risk of arrhythmic complications has been shown in a number of studies in the literature, as for instance [65]. In [66] 24-hour Holter recordings of post-MI patients are investigated by using the method described in [63]. The authors concluded that $Q T / R R$ analysis can be used to assess the efficacy of antiarrhythmic drugs.

2) $Q T$ variability: Other factors apart from HR contribute to QT modulation and their study has been suggested to provide clinically relevant information [67]. In addition to ANS action on the SA node, the direct ANS action on ventricular myocardium also alters repolarization and, thus, 
the QT interval [68]. Elucidation of the direct and indirect effects of ANS activity on QT may help assessing arrhythmia susceptibility [69].

QT variability (QTV) refers to beat-to-beat fluctuations of the QT interval and can be quantified in the time or the frequency domain. QTV is usually adjusted by HR variability (HRV) to assess direct ANS influence on the ventricles. In [70] QT variations out of proportion to HR variations were assessed by considering the following log-ratio index:

$$
Q T V I=\log _{10}\left[\frac{Q T_{v} / Q T_{m}^{2}}{H R_{v} / H R_{m}^{2}}\right],
$$

where $Q T_{m}$ and $Q T_{v}$ denote mean and variance of the $\mathrm{QT}$ series and $H R_{m}$ and $H R_{v}$ denote mean and variance of the HR series. In [71] QTV was evaluated by standard time domain indices like SDNN, RMSSD or pNN50, applied to the QT series; also, QTV was evaluated in the frequency domain by computing the total power as well as the power in different frequency bands. Other studies have assessed beat-tobeat variations in the shape or duration of ECG repolarization. In [72] repolarization morphology variability was computed by measuring the correlation between consecutive repolarization waves; in [73] a wavelet-based method was proposed to quantify repolarization variability both in amplitude and in time; in [74] time domain measures that quantified variability of the QT interval and of the T wave complexity were computed, with complexity assessed using PCA.

Other approaches to assess repolarization variability use parametric modeling [42], [75], [76]. While in [76] Porta et al. investigate variability of the RT interval, in [42] Almeida et al. explore QTV, and in [75] the variability from the R peak to the $\mathrm{T}$ wave end (RTe) is considered. The use of RT instead of QT avoids the need to determine the end of the T wave, which is usually considered to be problematic. However, due to the fact that the RT interval is shorter than the QT interval, its variability is much reduced and, more importantly, the information provided by RT variability and QTV has been shown to be different in certain populations, such as in patients with cardiovascular diseases [77]. In relation to this, recent studies in the literature have shown that the interval between the apex and end of the $\mathrm{T}$ wave possesses variability that is independent from HR and which can provide clinically useful information to be used for arrhythmic risk stratification [78]. The methodology described in [42] considers a linear parametric model to quantify the interactions between QTV and HRV, being applicable under steady-state conditions. The type of environments for analysis is, thus, substantially different from those considered in section III-C1, in which QT interval adaptation was investigated after possibly large HR changes. In [42] as much as $40 \%$ of QTV was found not to be related to HRV in healthy subjects. However, it should be noted that nonlinear effects were not considered in the analysis.

Increased repolarization variability has been reported under conditions predisposing to arrhythmic complications. Using the above described QTVI index, elevated variability has been reported in patients with dilated cardiomyopathy and in patients with hypertrophic cardiomyopathy, as compared with age-matched controls [79]. In [71] increased QTV in hyper- trophic cardiomyopathy patients was also found using standard time and frequency domain variability indices. Additionally, higher levels of repolarization variability (either in shape or duration) were found in LQTS patients [73], [74].

In patients presenting for electrophysiological testing, QTVI was significantly higher in the subgroup of those who had aborted SCD or documented VF [80]. In [81] increased QTVI was shown to be an indicator of risk for developing arrhythmic events (VT or VF) in post-MI patients. The association between increased repolarization variability and risk for VT/VF was also shown in [80] for post-MI patients with severe left ventricular (LV) dysfunction. In [75] an index quantifying autonomic control of HR and RTe was shown to separate symptomatic LQTS carriers from asymptomatic ones and controls.

Investigating the causes and modulators of the clinically observed, and eventually measured, temporal and spatial variability in ventricular repolarization is a challenging goal. The use of combined experimental and computational approaches can be a useful tool for such investigations [82]. In [83], [84] and [85] it was hypothesized that fluctuations in ionic currents caused by stochasticity in ion channel behavior contribute to variability in cardiac repolarization, particularly under pathological conditions. Also it was postulated that electrotonic interactions through intercellular coupling act to mitigate spatiotemporal variability in repolarization dynamics in tissue, as compared to isolated cells. The approaches taken in [83], [84] and [85] combine experimental and computational investigations in human, guinea pig and dog. Multiscale stochastic models of ventricular electrophysiology are used, bridging ion channel numbers to whole organ behavior. Results show that under physiological conditions: i) stochastic fluctuations in ion channel gating properties cause significant beat-tobeat variability in APD in isolated cells, whereas cell-to-cell differences in channel numbers also contribute to cell-to-cell APD differences; ii) in tissue, electrotonic interactions mask the effect of current fluctuations, resulting in a significant decrease in APD temporal and spatial variability compared to isolated cells. Pathological conditions resulting in gap junctional uncoupling or a decrease in repolarization reserve uncover the manifestation of current noise at cellular and tissue level, resulting in enhanced ventricular variability and abnormalities in repolarization such as afterdepolarizations and alternans.

Also it is worth noting that temporal QT interval variations may differ between recording leads due to the presence of local repolarization heterogeneity in the ECG signals. Leadspecific respiration effects or other types of noises can also have an effect. Respiration may influence QTV through APD modulation in ventricular myocytes [86], in particular during respiratory sinus arrhythmia [87] and by measurement artefacts in single ECG leads due to cardiac axis rotation, which can be compensated for by using careful methodological designs where the rotation angles introduced by respiration are taken into account [51]. Ventricular repolarization is also modulated by e.g. mechanoelectrical feedback in response to changes in ventricular loading [88]. 
D. ECG markers for characterization of spatio-temporal repolarization dispersion

1) T wave alternans: TWA, also referred to as repolarization alternans, is a cardiac phenomenon consisting on as a periodic beat-to-beat alternating change in the amplitude or shape of the ST-T complex, see Fig. 10.
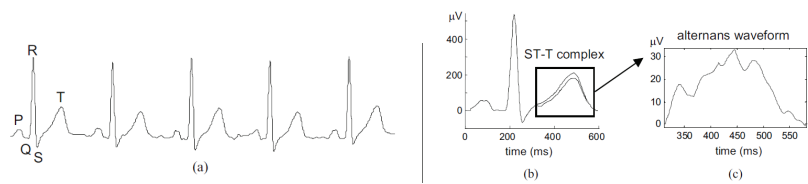

Fig. 10. An example of $\mathrm{T}$ wave alternans. The alternating behavior between two different $\mathrm{T}$ wave morphologies is particularly evident when all $\mathrm{T}$ waves are aligned in time and superimposed, as displayed on the middle panel (b). In (c) the alternanting waveform is amplified. Adapted from [89].

Although macroscopic TWA had been sporadically reported since the origins of electrocardiography, it was not until the generalization of computerized electrocardiology that it was possible to detect and quantify subtle TWA at the level of several microvolts [90]. Since then, TWA has been shown to be a relatively common phenomenon, usually associated with electrical instability. Therefore, it has been proposed as an index of susceptibility to ventricular arrhythmias.

The presence of TWA has been widely validated as a marker of SCD risk. A comprehensive review on physiological basis, methods and clinical utility of TWA can be found in [91], while the ionic basis of TWA has already been presented in section II-B1.

In most patients, increased HR is necessary to elicit TWA. Accordingly, measurement and quantification of TWA usually require the elevation of HR in a controlled way (usually by pacing or most commonly during exercise or pharmacological stress tests). It is interesting to note that unspecific TWA has been found at high HR in healthy subjects [92]. Thus, in order to be considered as an index of increased risk of SCD, it is usually considered that TWA should be present at HR below 110-115 bpm.

From the signal processing viewpoint, TWA analysis should be considered a joint detection-estimation problem [90]. The presence or absence of this phenomenon (i.e., a detection problem) is often the only information considered and most studies consider just the presence of TWA, regardless of its magnitude, as a clinical index. However, the magnitude of the observed TWA (i.e., an estimation problem) may also be relevant, as it has been shown that increasing TWA magnitude is associated with higher susceptibility to SCD [93]. Patterns of variation in the TWA magnitude can be seen in three domains: the distribution of alternans within the ST-T segment, the time course of TWA and the distribution of TWA in the different recorded leads.

The distribution of alternans within the repolarization interval is normally overlooked, as a global measurement for the whole ST-T segment (e.g. the maximal or the average TWA amplitude) is usually given. However, some authors have quantified the location of TWA, finding that it was more specific for inducible VT when it was distributed later in the ST-T segment [94]. Early TWA has been associated with acute ischemia, although different locations have been noticed as a function of the occluded artery [95]. Recent works have also focused on the delay of the alternant wave with respect to the $\mathrm{T}$ wave, defining a physiological range for this delay [96].

As TWA is a transient phenomenon, it must be quantified locally, which is usually done using an analysis window with a fixed width in beats. The time course of TWA can be tracked by moving the analysis window. In stress tests, changes in TWA amplitude are usually determined by changes in HR. Therefore, the TWA time course is usually related with HR changes. In stress tests, a set of rules involving the HR at which TWA appears and the episode duration has been proposed to determine the outcome of the TWA test [97]. The time course of TWA magnitude with respect to the onset of ischemia and reperfusion has been studied in the first minutes of acute ischemia, both in a human model (with ischemia induced by PCI) [95] and in animals [98]. However, at present, whether temporal patterns in TWA can be clinically useful for risk stratification is unknown [99].

The TWA magnitude distribution among the different leads have been studied during acute ischemia [95] and in postMI patients [100], showing different patterns according to the affected region of the myocardium, with higher amplitude TWA measured in leads close to the diseased areas.

a) Single-lead alternans detection: A general model for TWA analysis represents the ST-T complex of the $i$ th beat in the $l$ th lead as

$$
x_{i, l}(n)=s_{i, l}(n)+\frac{1}{2} a_{i, l}(n)(-1)^{i}+v_{i, l}(n), n=0, \ldots, N-1
$$

where $s_{i, l}(n)$ is the average ST-T complex, $a_{i, l}(n)$ is the alternant wave, and $v_{i, l}(n)$ is a noise term. Assuming that both $s_{i, l}(n)$ and $a_{i, l}(n)$ vary smoothly from beat to beat, the average ST-T complex can be easily cancelled out just by subtracting to each beat the ST-T of the previous beat $y_{i, l}(n)=x_{i, l}(n)-x_{i-1, l}(n)$, which is, according to the model $y_{i, l}(n)=a_{i, l}(n)(-1)^{i}+w_{i, l}(n)$, with $w_{i, l}(n)=$ $v_{i, l}(n)-v_{i-1, l}(n)$.

As described before, an analysis window must be defined for TWA analysis, assuming that the TWA wave is essentially constant within the beats included and shifting the window to cover the whole available signal. Let us consider a window of $K$ beats. For each possible position of the window (e.g., when centered at the $j$ th beat) the TWA analysis algorithm must decide whether TWA is absent $\left(a_{j, l}(n)=0\right.$ for every $\left.n\right)$ or present $\left(a_{j, l}(n) \neq 0\right)$ in the signal. This is usually done by computing a detection statistic $Z_{j, l}$ quantifying the likelihood that there is indeed TWA in the signal and comparing it to some threshold. Besides, algorithms usually provide either an estimate $\hat{a}_{j, l}(n)$ of the alternant wave present in each lead of the signal or a global TWA magnitude $A_{j, l}$, as, for instance, the RMS of $\hat{a}_{j, l}(n)$. Note that the time course and lead-distribution of TWA is given by the variations of $Z_{j, l}$ and $A_{j, l}$ with the beat and lead indices, respectively.

The reader can find in [90] a comprehensive methodological review of the techniques that have been proposed for TWA detection and estimation. According to the TWA analysis approaches, the authors classify all schemes as equivalent to one of these signal processing techniques: the short-term 
Fourier transform (STFT), count of sign-changes and nonlinear filtering.

Methods of the first class are based on the classical windowed Fourier analysis, applied to beat-to-beat series of synchronized samples within the ST-T complex. Evaluating the STFT at 0.5 cycles per beat, we obtain the TWA component at the $n$-th sample.

$$
z_{j, l}(n)=\sum_{i=-\infty}^{\infty} x_{i, l}(n) w(i-j)(-1)^{i} .
$$

As shown in [90], this process is equivalent to apply a linear high-pass filter to the beat-to-beat series with subsequent demodulation. The width of the analysis window $w(i)$ expresses the compromise between accuracy and tracking ability of the algorithm. The linearity of the STFT makes these methods to be quite sensitive to artifacts or impulsive noise in the beatto-beat series. The widely used spectral method [101] belongs to this class. It estimates additional frequency components to have an estimation of the noise level. For detection, a TWA ratio is defined and compared to a threshold. This makes the method less sensitive to variations in the noise level, thus reducing the risk of false alarms.

The second class includes methods quantifying TWA according to the analysis of sign-changes in the detrended beatto-beat series [90]. These methods are quite robust against impulsive noise and artifacts, but are easily affected by the presence of other non-alternant components. The amplitude information is also lost when using these techniques.

Methods in the third class use nonlinear time domain approaches instead of the linear filtering or Fourier-based techniques. The modified moving average method estimates the ST-T complex patterns for the odd and even beats, using a recursive moving average whose updating term is modified by a nonlinear limiting function [102]. TWA is then estimated at each beat as the difference between the odd and even estimated ST-T complexes. The main difference with respect to linear techniques arises when there are abrupt changes in the waves, due to noise, artifacts or abnormal beats: then the nonlinear function keeps the effect on the TWA estimate bounded. However, the method is sensitive to noise level changes, as it does not consider adaptation to the noise level.

The Laplacian likelihood ratio (LLR) uses a statistical model approach, considering a signal model similar to (30), where the noise term is modelled as a zero-mean Laplacian random variable with unknown variance. The generalised likelihood ratio test (GLRT) and the maximum likelihood estimate (MLE) are used, respectively, for TWA detection and estimation at each position of the analysis window [90]. The assumption of a heavy-tailed noise distribution makes the method more robust to outiers in the beat-to beat series. The MLE of the alternant wave is the median-filtered demodulated beat-to-beat series [95]

$$
\hat{a}_{j, l}(n)=\operatorname{median}\left\{y_{i, l}(n)(-1)^{i}\right\}_{i \in W_{j},}
$$

where $W_{j}$ is the analysis window centered at beat $j$. The GLRT statistic is

$$
Z_{j, l}=\frac{\sqrt{2}}{\hat{\sigma}_{j, l}} \sum_{n=0}^{N-1}\left(\sum_{i \in W_{j}}\left|y_{i, l}(n)\right|-\left|y_{i, l}(n)-\hat{a}_{j, l}(n)(-1)^{i}\right|\right),
$$

where $\hat{\sigma}_{j, l}=\frac{1}{\sqrt{2} N L} \sum_{n=0}^{N-1} \sum_{i \in W_{j}}\left|y_{i, l}(n)-\hat{a}_{j, l}(n)(-1)^{i}\right|$ is an estimation of the noise standard deviation. It can be proved that the probability of a false alarm with this scheme does not depend on the noise level. This TWA detector has also been tested and successfully applied on invasive EGM signals [103].

Although all these methods are usually applied on a leadby-lead basis, works using a multi-lead strategy suggest that improved performance can be achieved by jointly processing all the available leads, taking advantage of the different interlead correlation of TWA and noise components [104].

b) Multi-lead alternans detection: Methodological approaches for multi-lead alternans detection have been presented in the literature, which integrate all the available leads in such a way that the alternans is reinforced, making subsequent TWA detection more robust.

We present here two multi-lead approaches [104], one based on $\pi \mathrm{CA}$ (multi- $\pi C A)$ [105] and another one based on PCA (multi-PCA) [89]. Both approaches follow a general scheme whose main stages are: preprocessing, signal transformation, TWA detection, signal reconstruction and TWA estimation (Fig. 11). The difference between multi-PCA and multi- $\pi C A$ is the way to perform the signal transformation (and reconstruction).

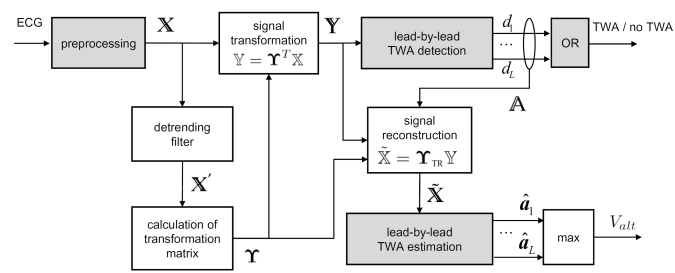

Fig. 11. Block diagram of the general TWA multi-lead analysis scheme. Blocks in bold line are the ones used in the single-lead scheme, in which $\mathbb{Y}=\mathbb{X}=\tilde{\mathbb{X}}$. Adapted from [104].

Signal preprocessing: After determining QRS positions and removing baseline wander, the ECG signal can be low-pass filtered and decimated to a sampling frequency of $F_{s} \geq 30$, thus removing off-band noise while keeping TWA frequency components [106]. In each beat, an interval of $350 \mathrm{~ms}$ (the ST-T complex, corresponding to the $N$ samples referred in (30)) is selected for TWA analysis. In vector notation, the ST$\mathrm{T}$ complex presented in eq. (30) is denoted as

$$
\mathbf{X}_{i}=\left[\mathbf{x}_{i, 1}, \ldots, \mathbf{x}_{i, L}\right]^{T}, \quad \mathbf{x}_{i, l}=\left[x_{i, l}(0), \ldots, x_{i, l}(N-1)\right]^{T}
$$

where for each beat $i$, matrix $\mathbf{X}_{i}$ is built with the ST-T complexes from all leads $\left(\mathbf{x}_{i, l}, l=1, \ldots, L\right)$.

The data matrix $\mathbb{X}$ is then constructed by concatenating the matrices $\mathbf{X}_{i}$ for the $K$ beats in the analysis window,

$$
\mathbb{X}=\left[\begin{array}{llll}
\mathbf{X}_{0} & \mathbf{X}_{1} & \ldots & \mathbf{X}_{K-1}
\end{array}\right]
$$

and finally the matrix $\mathbb{X}^{(m)}$ is constructed as

$$
\mathbb{X}^{(m)}=\left[\begin{array}{llll}
\mathbf{X}_{m} & \mathbf{X}_{m+1} & \ldots & \mathbf{X}_{m+K-1}
\end{array}\right]
$$

which is equivalent to $\mathbb{X}$, but shifting the analysis window $m$ beats forward.

Signal transformation: The aim of this stage is to apply a linear transformation to the signal $\mathbb{Y}=\boldsymbol{\Upsilon}^{T} \mathbb{X}$ that improves the 
detectability of TWA by exploiting the information available in the multi-lead ECG (see Fig. 12).
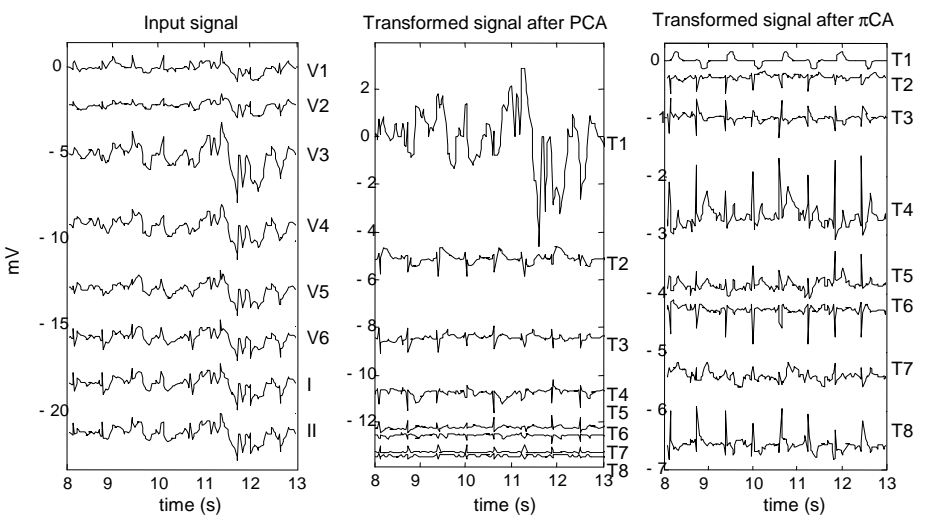

Fig. 12. a)(a) Eight independent leads) of a real 12-lead ECG where TWA of $200 \mu \mathrm{V}$ was artificially added. TWA is invisible to the naked eye due to noise and artifacts. (b) Signal in (a) after PCA transformation. TWA is now visible in the transformed lead T2 through exaggerated oscillations in the amplitude of the $\mathrm{T}$ wave. (c) Signal in (a) after $\pi \mathrm{CA}$ transformation. TWA is clearly visible in transform lead T1. Reproduced from [104].

In order to obtain a suitable transformation matrix $\Upsilon$ the average ST-T complexes are canceled out by subtracting the previous complex from each complex $\mathbf{x}_{i, l}^{\prime}=\mathbf{x}_{i, l}-\mathbf{x}_{i-1, l}$. These detrended beats $\mathbf{x}_{i, l}^{\prime}$ are used to build the matrices $\mathbb{X}^{\prime}$ and $\mathbb{X}^{(m)^{\prime}}$ as in (35) and (36). Note that $\mathbb{X}^{\prime}$ and $\mathbb{X}^{(m)^{\prime}}$ now contain $K-1$ beats. The transformation matrix $\Upsilon$ can be obtained as described in the following paragraphs. Note that considering the identity transformation matrix, the multi-lead scheme reduces to a single-lead scheme, handling each lead independently throughout the detection/estimation process.

Principal Component Analysis: The detrended signal $\mathbf{x}_{i, l}^{\prime}$ is a zero-mean random vector whose spatial correlation can be estimated as

$$
\mathbf{R}_{\mathbb{X}^{\prime}}=\frac{1}{(K-1) N} \mathbb{X}^{\prime} \mathbb{X}^{\prime} T
$$

The PCA transformation matrix is obtained by solving the eigenvector equation for matrix $\mathbf{R}_{\mathbb{X}^{\prime}}$

$$
\mathbf{R}_{\mathbb{X}^{\prime}} \Upsilon=\Upsilon \Lambda
$$

where $\boldsymbol{\Lambda}$ is the diagonal eigenvalue matrix, where eigenvalues are sorted in descending order, and $\Upsilon$ is the corresponding orthonormal eigenvector matrix. The transformation defined by matrix $\Upsilon$ is then applied to the original data matrix $\mathbb{X}$ to obtain the transformed matrix

$$
\mathbb{Y}=\Upsilon^{T} \mathbb{X}
$$

whose $l$ th row ( $l$ th transformed lead) contains the $l$ th principal component of $\mathbb{X}$. Fig. 12(b) shows the PCA transformation of the input signal in (a).

Periodic Component Analysis: The aim of this technique is to find a linear combination of the available leads $\mathbf{y}_{i}^{T}=$ $\mathbf{w}^{T} \mathbb{X}_{i}^{\prime}$ enhancing the 2-beat periodicity corresponding to TWA (equivalent to a frequency of 0.5 cycles per beat). The desired weight vector is obtained by minimizing

$$
\epsilon(\mathbf{w}, 2)=\frac{\sum_{i=0}^{K-1}\left\|\mathbf{y}_{i+2}^{\prime}-\mathbf{y}_{i}^{\prime}\right\|^{2}}{\sum_{i=0}^{K-1}\left\|\mathbf{y}_{i}^{\prime}\right\|^{2}}
$$

As shown in [105], (40) can be rewritten as

$$
\epsilon(\mathbf{w}, 2)=\frac{\mathbf{w}^{T} \mathbf{A}_{\mathbb{X}^{\prime}}(2) \mathbf{w}}{\mathbf{w}^{T} \mathbf{R}_{\mathbb{X}^{\prime}} \mathbf{w}}
$$

where $\mathbf{R}_{\mathbb{X}^{\prime}}$ is defined in (37) and $\mathbf{A}_{\mathbb{X}^{\prime}}(2)$ is the spatial correlation of $\left(\mathbb{X}^{(2)^{\prime}}-\mathbb{X}^{\prime}\right)$, which can be estimated as

$$
\mathbf{A}_{\mathbb{X}^{\prime}}(2)=\frac{1}{(K-1) N}\left(\mathbb{X}^{(2)^{\prime}}-\mathbb{X}^{\prime}\right)\left(\mathbb{X}^{(2)^{\prime}}-\mathbb{X}^{\prime}\right)^{T}
$$

The weight vector $\mathbf{w}$ minimizing (41) is the generalized eigenvector associated with the smallest generalized eigenvalue of the matrix pair $\left(\mathbf{A}_{\mathbb{X}^{\prime}}(2), \mathbf{R}_{\mathbb{X}^{\prime}}\right)$ [105]. Generalizing this result, the complete transformation matrix $\Upsilon$ can be defined as the matrix whose columns are the generalized eigenvectors of $\left(\mathbf{A}_{\mathbb{X}^{\prime}}(2), \mathbf{R}_{\mathbb{X}^{\prime}}\right)$ ordered in ascending order of magnitude of their associated eigenvalues. In this way, the first row of the transformed data matrix $\mathbb{Y}=\mathbf{\Upsilon}^{T} \mathbb{X}$ contains the most periodic combination of leads (i.e. it is the transformed lead where TWA - if present - is more easily detectable). Fig. 12(c) shows the $\pi \mathrm{CA}$ transformation of the signal in (a).

TWA detection: After transforming the signal, single-lead TWA detection is performed individually on each row of the transformed signal $\mathbb{Y}$ applying the LLR method [95] described in equation (33). As a result, the decision for lead $l$ will be $d_{l}=1$ if TWA is present in the $l$ th transformed lead, and $d_{l}=0$ otherwise. The multi-lead TWA detection is positive if detection is at least positive in one transformed lead ('OR' block in Fig. 11), and negative otherwise.

Signal reconstruction: To allow a better clinical interpretation, it is important to have TWA measured in the original lead set. For that purpose, a reconstructed signal in the original leads can be obtained, taking only those transformed leads where TWA was detected to be present. A matrix $\Upsilon_{\mathrm{TR}}$ is obtained by replacing with zeros the columns of $\Upsilon$ corresponding to leads without TWA $\left(d_{l}=0\right)$, thus discarding non-alternant components. The reconstructed signal is then obtained as

$$
\tilde{\mathbb{X}}=\Upsilon_{\mathrm{TR}} \mathbb{Y}
$$

TWA estimation and clinical indices: The MLE for Laplacian noise (32) can be applied individually to each reconstructed lead $l$ to estimate the TWA waveform, $\hat{a}_{j, l}(n)$. A global amplitude of TWA $\left(A_{j, l}\right)$ can finally be defined as the RMS of $\hat{a}_{j, l}(n)$.

From these values clinical indices need to be derived, particularly when long term recordings are processed [107]. Some indices reflect the average amplitude of TWA and others quantify the maximum TWA amplitude in specific segments under study. An index of the former type is described as index of average alternans (IAA) and their corresponding versions after restriction to a specific interval for a $H R$ value $X$ are described as HRrestricted indices of average alternans $\left(\operatorname{IAA}_{X}\right)$, all of them proposed in [107]. IAA was computed as the average of all $V_{\text {alt }}$ measured in 128-beat data windows during the 24-hour period, thus reflecting the average TWA activity. For instance, a 24-hour ECG presenting TWA for 5\% of the time with an amplitude of $60 \mu \mathrm{V}$ would have an IAA=3 $\mu \mathrm{V}$. IAA $_{X}$ values $(\mathrm{X}=\{70,80,90,100,110\}$ beats/min $)$ 
were computed in a similar way, but considering only those $V_{\text {alt }}$ measured in segments with average HR ranging from $\mathrm{X}-10$ to $\mathrm{X}$ beats $/ \mathrm{min}$. Therefore $\mathrm{IAA}_{90}$ reflects the average TWA activity at HR between 80 and 90 beats/min in 24 hours. Clinical studies where these multi-lead techniques have already being applied can be found in [107] and [108].

Finally, a note of caution: in stress test recordings, the running/pedaling rate can overlap with the alternans rate when the HR doubles the running/pedaling rate [109], e.g a cadence of $65 \mathrm{rpm}$ could create alternans-like artifacts at a HR of 130 bpm due to the synchronous body movements, which could be erroneouly interpreted as electrical alternans [110].

c) T wave alternans in view of the biophysical modeling: ECG repolarization alternans, as mentioned before, can result from AP instability, which can be measured as fluctuations in the duration of the AP. This phenomenon can be introduced in the biophysical modeling already introduced in section II-C to gain insight into how this AP duration fluctuation is reflected on the $\mathrm{T}$ wave morphology and thus improve interpretation or design of TWA detection [111].

In the model in (5), $\rho_{m}=\bar{\rho}+\Delta \rho_{m}$, the dispersion heterogeneity $\Delta \rho_{m}$ can be made explicitly dependent on $i$ th beat and further split into

$$
\Delta \rho_{m}(i)=\vartheta_{m}+\varphi_{m}(i)+(-1)^{i} \frac{\delta_{m}}{2},
$$

where $\vartheta_{m}$ describes the spatial variation of the repolarization times for a given subject (at a given HR), $\varphi_{m}(i)$ reflects the slight, physiological beat-to-beat differences in repolarization times and $\delta_{m}$ accounts for alternans in the repolarization times occurring between even and odd beats in node $m$.

The value of $\vartheta_{m}$ can be estimated for a given beat by solving the inverse electrocardiographic problem. $\varphi_{m}(i)$ can be seen as a noise term and approximated by a set of independent zero-mean normal random variables, with standard deviation $\sigma_{\varphi}$. For simplicity, in the short term, the standard deviation $\sigma_{\varphi}$ is considered to be invariant with time and across myocardial regions. The term $\delta_{m}$ is also assumed to be constant within the analysis window [111].

Note that changes in HR affect the terms in this model [111]. When HR increases, the APD shortens and $\bar{\rho}$ moves closer to the onset of the beat, but it is also known that the $\mathrm{T}$ wave becomes more symmetrical with increased HR, including its area, while its amplitude decreases [112]. This would suggest modifying $\Delta \rho_{m}(i)$. However, other studies have shown that the T-peak to T-end interval is mostly independent from HR at rest [112], even if contradictory results have also been published [113], and that the maximal difference among APDs measured in isolated Langendorff- perfused rabbit hearts [114] did not change significantly throughout the range of paced steady-state cycle lengths, suggesting a minor effect of the HR on $\Delta \rho_{m}(i)$. Possibly the two effects coexist: (i) a small reduction of the spatial dispersion of repolarization with increasing HR and (ii) a modification with HR of the shape of the TMP in phases 2 and 3 [111].

According to the first order approximation in equation (8),
$\mathbf{X} \approx \mathbf{w}_{1} \mathbf{t}_{d}^{T}$, the $\mathrm{T}$ wave for lead $l$ and beat $i$, is:

$$
x_{i, l}(t)=-t_{d}(t) \sum_{m=1}^{M} A_{l, m} \Delta \rho_{m}(i) .
$$

By computing the difference between two consecutive $\mathrm{T}$ waves

$$
\begin{aligned}
x_{i+1, l}(t)-x_{i, l}(t) & =-t_{d}(t) \sum_{m=1}^{M} A_{l, m}\left[\Delta \rho_{m}(i+1)-\Delta \rho_{m}(i)\right] \\
& =-t_{d}(t) \sum_{m=1}^{M} A_{l, m}\left[\varphi_{m}(i+1)-\varphi_{m}(i)+\delta_{m}\right]
\end{aligned}
$$

where the assumption is made that the dominant $\mathrm{T}$ wave does not change between the two beats. Repeating the process on successive couples of beats and averaging the results, the following expression is obtained:

$$
E\left[x_{i+1, l}(t)-x_{i, l}(t)\right]=-t_{d}(t) \sum_{m=1}^{M} A_{l, m} \delta_{m} .
$$

Therefore, under this model, TWA depends on $\delta_{m}$ (alternans in the myocytes' repolarization times), but also on the dominant $\mathrm{T}$ wave $t_{d}(t)$. If regional alternans is considered, $\delta_{m} \neq 0$ only for several nodes, while in the case of global alternans this would occur in each node $m$. In both situations, and given a set of $\delta_{m}$, TWA also depends on the matrix $\mathbf{A}$ and thus varies for different subjects and leads [111].

2) Repolarization dispersion by T-peak to T-end interval dynamics: In section II-B1 we already mentioned that the APDR curve, and the increase in its dispersion, is related to greater propensity to suffer arrythmias (Fig. 13), therefore providing potentially relevant information for ventricular arrhythmic risk stratification [25], [115]. Heterogeneities in the ventricle lead to non-uniform restitution properties, which makes APDR curves present spatial variations [116].

Quantification of APDR dispersion requires invasive techniques [117]. A method has been proposed in [20] to indirectly estimate dispersion in the dynamic APDR slopes by evaluating the relationship between the $T_{p e}$ and the $R R$ interval under different stationary conditions. The $T_{p e}$ interval reflects differences in the time for completion of repolarization at different ventricular regions. While some studies postulate that $T_{p e}$ measures transmural dispersion of repolarization [48], other studies claim that $T_{p e}$ includes additional heterogeneities, such as apico-basal ones [49].

For ECG segments of stable HRs, the index $\Delta \alpha$ (see Fig. 5 , bottom-left) is defined as:

$$
\widehat{\Delta \alpha}^{\mathrm{ECG}_{\mathrm{s}}}=\frac{\Delta T_{p e}^{d y n}}{\Delta R R}
$$

where " $E C G_{s}$ " indicates stable ECG segments, as required in the dynamic protocol, at two different $R R$ intervals, $\Delta$ at the left hand side of (48) refers to a difference of restitution slopes occurring at two regions, while both $\Delta$ at the right hand side refer to beat interval differences associated with two $R R$ levels [20].

For ECG segments of unstable HR, computation of the index $\Delta \alpha$ includes a methodology to compensate for the $T_{p e}$ hysteresis lag after $R R$ changes. The model used to estimate $T_{p e}$ hysteresis is the same presented in Fig. 9 but replacing the $Q T$ interval with the $T_{p e}$ interval. The $R R$ and $T_{p e}$ series of 

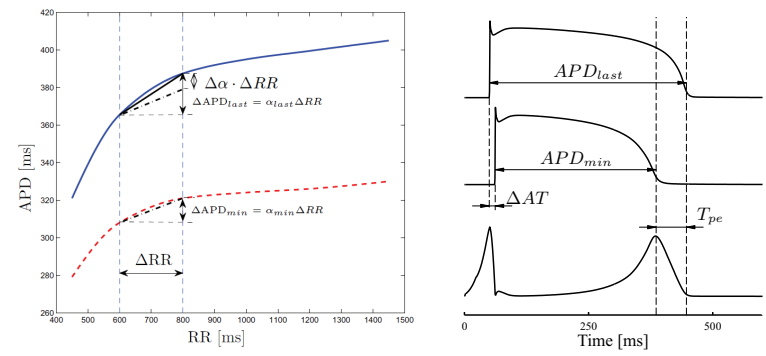

Fig. 13. Left panel: Dynamic restitution curves of two cells representing APD as function of RR. Right panel: Representation of the $T_{p e}$ interval in terms of APDs and delay of activation times $(\Delta A T)$. Adapted from [20].

each recording are interpolated and resampled to a sampling frequency of $f_{s}=1 \mathrm{~Hz}$. The length $N$ of vector $\mathbf{h}$ was set to 150 samples that correspond to 150 seconds, which widely exceeds the $T_{p e}$ memory lag [20]. Details on the optimization algorithm used for parameter identification can be found in [118].

After $\mathbf{h}$ and $g_{k}(., \mathbf{a})$ have been optimized, $z_{\mathrm{RR}}(n)$ can be used as a surrogate of the running $R R$ series that would generate a truly stationary period in the running repolarization interval $T_{p e} .\left[T_{p e}(i), z_{\mathrm{RR}}(i)\right]$ represents the $T_{p e}$ interval and the surrogate for the $R R$ interval after $T_{p e}$ hysteresis compensation. The estimate of restitution dispersion derived in (48) for stable HR segments can be replaced with the following equation:

$$
\widehat{\Delta \alpha}^{\mathrm{ECG}}=\left.\frac{\partial T_{p e}}{\partial z_{\mathrm{RR}}}\right|_{z_{\mathrm{RR}}=\bar{z}_{\mathrm{RR}}}=\left.\frac{\partial g_{k}\left(z_{\mathrm{RR}}, \mathbf{a}\right)}{\partial z_{\mathrm{RR}}}\right|_{z_{\mathrm{RR}}=\bar{z}_{\mathrm{RR}}}
$$

with $g_{k}$ being the nonlinear function represented in Figure 9. The above expression has the advantage of avoiding the need for stationary ECG segments. The superindex "ECG ${ }_{c}$ " indicates that the quantification is done by compensating for the $T_{p e}$ memory lag. This estimate is a robust alternative to $\widehat{\Delta \alpha}{ }^{\mathrm{ECG}_{\mathrm{s}}}$ (see Fig. 5 bottom-left). In (49), the derivative is evaluated at the mean $z_{\mathrm{RR}}$ value, $\bar{z}_{\mathrm{RR}}$, of the analyzed ECG recording.

Electrophysiological modeling and simulation has been used to evaluate $\widehat{\Delta \alpha}^{\mathrm{ECO} \sigma_{\mathrm{S}}}$ and $\widehat{\Delta \alpha}{ }^{\mathrm{ECG}}$ as measurements of APDR slope dispersion at tissue level (see Fig. 5, right) [20]. Electrical propagation in a left ventricular $2 \mathrm{D}$ tissue has been simulated using a human ventricular AP model [14] and numerical integration as described in [119]. The 2D tissue slice covers base to apex and endocardial to epicardial distances, as illustrated in Fig. 5, top right panel. For details about the simulation see [20]. APDR curves are computed following dynamic pacing at different $R R$ intervals. Simulated APDR slope dispersion is denoted by $\Delta \alpha^{\mathrm{SIM}}$, which is computed as:

$$
\Delta \alpha^{\mathrm{SIM}}=\frac{\partial A P D_{\text {last }}^{\text {dyn }}}{\partial R R}-\frac{\partial A P D_{\text {min }}^{\text {dyn }}}{\partial R R}
$$

where $A P D_{\min }$ corresponds to the cell with the minimum APD among those which are repolarizing at the $T$ wave peak instant (time instant when the maximum repolarization gradient sum occurs) and $A P D_{\text {last }}$ is the APD of the last cell to repolarize. Estimations of $\Delta \alpha^{\text {sim }}$ are computed from pseudoECGs using (48). Each pseudo-ECG evaluates the extracellular potential at a sensor position (Fig. 5, bottom-right) [120]. The corresponding estimation is:

$$
\widehat{\Delta \alpha}^{\mathrm{PECG}}=\frac{\partial T_{p e}^{d y n}}{\partial R R}
$$

where $T_{p e}^{d y n}$ represents the $T_{p e}$ interval measured from the pseudo-ECG under the dynamic protocol (see Fig. 14).

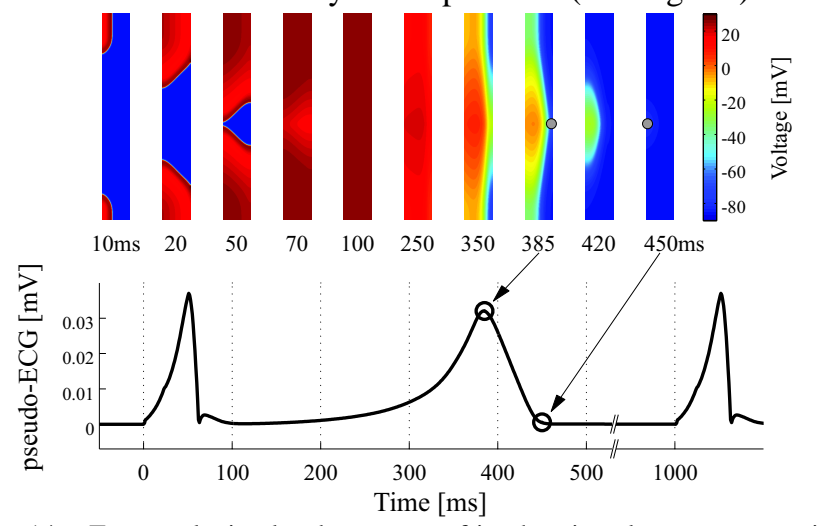

Fig. 14. Top panel: simulated sequence of isochronic voltage representation during steady-state pacing at $1000 \mathrm{~ms}$. The position of the two cells corresponding to $A P D_{\min }$, for the the $\mathrm{T}$ wave peak, and $A P D_{\text {last }}$, for the $\mathrm{T}$ wave end, are shown with a gray point. Bottom panel: derived pseudo-ECG. Reproduced from [20].

The value of the index $\Delta \alpha$ for risk stratification has been explored in different populations, as in patients who developed TdP after sotalol administration [121], in patients with hypertrophic cardiomyopathy [122] and in patients with heart failure [123], where the prognostic value of $\Delta \alpha$ could be corroborated in retrospective studies, and with the provided information being complementary to that obtained from other repolarization or autonomic indices measuring TWA (IAA parameter) or heart rate turbulence (HRT).

3) Repolarization dispersion by biophysical model parameter estimation: VRD can account for heterogeneities in the AP shape across the ventricular wall, but mainly reflects differences in the APD. Taking the biophysical model presented in section II-C as a starting point, a method has been proposed in [34] to quantify this dispersion. One of the goals is to show that VRD can be quantified by estimating the standard deviation of the time instants $\rho_{m}$ (named $s_{\vartheta}$ ). This dispersion includes two sources, one from the fact that AP activation times are not synchronous at all nodes (see Fig. 6) and the other one from the fact that APD is different at different sites (nodes). Since it is known that the dispersion in activation time instants is much smaller than the dispersion in the APDs, the variance in $\rho_{m}$ is assumed to be a valid surrogate for the dispersion of the repolarization times.

In [34] a procedure is developed based on a stochastic model for $\rho_{m}$. The $\mathcal{R} \mathcal{T}$ associated with the $m$ th node, introduced in equation (5), can be expressed as explicitely depending on beat $i$ :

$$
\rho_{m}(i)=\bar{\rho}(i)+\Delta \rho_{m}(i)=\bar{\rho}(i)+\vartheta_{m}+\varphi_{m}(i)
$$

where $\bar{\rho}(i)$ is the average $\mathcal{R} \mathcal{T}$, which may be different for each beat $i$, and $\Delta \rho_{m}(i) \ll \bar{\rho}(i)$ is the dispersion with respect to the average value (note that $\sum_{m=1}^{M} \Delta \rho_{m}(i)=0$ ). Although both terms are expected to depend on HR, the discussion in 
[34] is limited to the case of stationary HR. In this condition, $\Delta \rho_{m}(i)$ is split into two terms, $\vartheta_{m}$ and $\varphi_{m}(i)$, as shown in (52). On the one hand, $\vartheta_{m}$ describes the spatial variation of the $\mathcal{R} \mathcal{T}$ s considering the sequence of repolarization, starting from the apex of the ventricles. On the other hand $\varphi_{m}(i)$ accounts for the slight, physiological beat-to-beat differences in $\mathcal{R} \mathcal{T}_{\text {s. As }}$ it will be later discussed, it is reasonable to consider that, in general, $\varphi_{m}(i) \ll \vartheta_{m}$. If the distribution of the $\mathcal{R} \mathcal{T}$ s is affected by a pathological condition, this will be reflected in the model by a change in $\vartheta_{m}$, thus this is the main index to be measured, while $\varphi_{m}(i)$ can be seen as a noise term affecting the measurements at every beat and can be assumed to be independent of $m$ and normally distributed with variance $\sigma_{\varphi}^{2}(i)$ (see [34] for details). Also independence of $\varphi_{m}(i)$ for different sites $(m)$ and beats $(i)$ is assumed. If $\sigma_{\varphi}^{2}(k)$ is constant among beats (specially in stable HR and short time windows), the dependence on beat $i$ can be dropped and $\Delta \rho_{m}(i) \backsim \mathcal{N}\left(\vartheta_{m}, \sigma_{\varphi}^{2}\right)$.

Linking the above described modeling with the lead factors, $\mathbf{w}_{1}$ and $\mathbf{w}_{2}$, we have:

$$
w_{1}(l)=-\sum_{m=1}^{M} A_{l, m} \Delta \rho_{m}, w_{2}(l)=\frac{1}{2} \sum_{m=1}^{M} A_{l, m} \Delta \rho_{m}^{2} .
$$

By averaging in time, the mean and variance of $w_{1}(l)$ can be calculated as:

$$
E\left[w_{1}(l)\right]=-\sum_{m=1}^{M} A_{l, m} \vartheta_{m}, \operatorname{var}\left[w_{1}(l)\right]=\sigma_{\varphi}^{2} \sum_{m=1}^{M} A_{l, m}^{2} .
$$

Proceeding similarly with $w_{2}(l)$ and using the fact that $\sum_{m=1}^{M} A_{i, m}=0$, we have:

$$
\begin{gathered}
E\left[w_{2}(l)\right]=\frac{1}{2} \sum_{m=1}^{M} A_{l, m} \vartheta_{m}^{2} \\
\operatorname{var}\left[w_{2}(l)\right]=\frac{1}{4} \sum_{m=1}^{M} A_{l, m}^{2} \operatorname{var}\left[\Delta \rho_{m}^{2}\right] \\
=\frac{\sigma_{\varphi}^{4}}{2} \sum_{m=1}^{M} A_{l, m}^{2}+\sigma_{\varphi}^{2} \sum_{m=1}^{M} A_{l, m}^{2} \vartheta_{m}^{2}
\end{gathered}
$$

where the equality $E\left[\Delta \rho_{m}^{4}\right]=\vartheta_{m}^{4}+6 \vartheta_{m}^{2} \sigma_{\varphi}^{2}+3 \sigma_{\varphi}^{4}$ has been used. Defining the following ratio:

$$
\frac{\operatorname{var}\left[w_{2}(l)\right.}{\operatorname{var}\left[w_{1}(l)\right]}=\frac{\sigma_{\varphi}^{2}}{2}+\frac{\sum_{m=1}^{M} A_{l, m}^{2} \vartheta_{m}^{2}}{\sum_{m=1}^{M} A_{l, m}^{2}}
$$

and doing some additional algebraic calculations [34]

$$
\begin{aligned}
\sum_{m=1}^{M} A_{l, m}^{2} \vartheta_{m}^{2} & =\underbrace{\left(\sum_{m=1}^{M} \frac{\vartheta_{m}^{2}}{M}\right)}_{s_{\vartheta}^{2}}\left(\sum_{m=1}^{M} A_{l, m}^{2}\right) \\
& +\underbrace{\sum_{m=1}^{M}\left(A_{l, m}^{2}-\sum_{m^{\prime}=1}^{M} \frac{A_{l, m^{\prime}}^{2}}{M}\right)\left(\vartheta_{m}^{2}-\sum_{m^{\prime}=1}^{M} \frac{\vartheta_{m^{\prime}}^{2}}{M}\right)}_{C_{l}}
\end{aligned}
$$

the ratio can be calculated as

$$
\frac{\operatorname{var}\left[w_{2}(l)\right]}{\operatorname{var}\left[w_{1}(l)\right]}=\frac{\sigma_{\varphi}^{2}}{2}+s_{\vartheta}^{2}+\frac{C_{l}}{M} \frac{M}{\sum_{m=1}^{M} A_{l, m}^{2}} .
$$

Given that variability across time is supposed to be much smaller than the spatial dispersion $\sigma_{\varphi}^{2} \ll s_{\vartheta}^{2}$ and than $C_{l} / M$ can be seen as the covariance of the $l$ th row of matrix $\mathbf{A}$ with the spatial dispersion $\vartheta_{m}$, which are not coherent, and in addition equation (59) is normalized by the variance of the elements in the $l$ row of $\mathbf{A}$, the $\mathcal{V}$-index can be defined as:

$$
\mathcal{V}_{l}=\frac{\operatorname{std}\left[w_{2}(l)\right]}{\operatorname{std}\left[w_{1}(l)\right]} \approx s_{\vartheta}^{2} .
$$

This $\mathcal{V}$-index turns out to be independent of $l$ and represents the VRD measurement proposed in [34]. $\mathcal{V}_{l}$ needs to be estimated by calculating the lead factors $\mathbf{w}_{1}$ and $\mathbf{w}_{2}$, which can be done as explained in section II-C.

This modeling has several approximations and assumptions that may question to what extent relevant clinical information may be missed when computing the $\mathcal{V}$-index. In [124], with the aim to check for this, known dispersion was simulated in a tissue model. The biophysics-based $\mathcal{V}$-index was computed and the results compared to the simulated dispersion. It has been proved that when the assumptions are relatively well satisfied, the $\mathcal{V}$-index is a good approximation, but when the independence assumption between nodes and time is lost, high bias appears between estimation and reality. In some circumstances this can be overcome with some modifications of the $\mathcal{V}$-index presented in [124].

Clinical application of the index has already been explored in [125], where increased dispersion of the repolarization times, as measured by the $\mathcal{V}$-index, was found in patients with Chagas disease, being significantly correlated with the risk of death in a univariate survival analysis. Already in the initial study [34] it was shown that the index increases with sotalol administration, known to increase VRD. Later in [126], the $\mathcal{V}$ index was studied under moxifloxacin administration, showing a higher relative increase than the corrected QT interval.

The modeling here presented has been subsequently extended to analyze and correlate other $\mathrm{T}$ wave features with VRD, including the QT interval, $\mathrm{T}$ wave variability and others [40]. The $\mathcal{V}$-index is the one that results in the highest correlation to VRD. The QT interval is also highly correlated, with the drawback that it requires precise delineation of the $T$ wave end, which is a problematic technical problem.

\section{E. Summary of repolarization risk markers.}

The repolarization markers presented all along this review are summarized in the table I together with references to their physiological background and clinical evaluation.

TABLE I

SUMMARY OF RISK MARKERS, REFERENCES TO THEIR PHYSIOLOGICAL BACKGROUND AND CLINICAL EVALUATION.*AND REFERENCES THEREIN

\begin{tabular}{|l|c|c|}
\hline Marker class & Physiological background & Clinical evaluation \\
\hline B1. Repolarization intervals & {$[45],[46],[127]$} & {$[47]$} \\
\hline B2. T wave morphology & {$[46],[52],[55]$} & {$[47],[53],[54],[128]$} \\
\hline C1. QT adaptation to HR & {$[59],[60],[62],[61]$} & {$[63],[65],[66]$} \\
\hline C2. QT variability & {$[84],[68],[69],[82]$} & {$[70],[71],[72],[73]$} \\
& {$[83],[85],[42],[76]$} & {$[75],[77],[79],[80]$} \\
& {$[81]$} \\
\hline D1. T-wave alternans & {$[91],[92],[95],[98]$} & {$[91]^{*},[93],[107]$} \\
& {$[23],[24]$} & {$[108]$} \\
\hline D2. Tpe dynamics & {$[129],[130],[116],[117]$} & {$[121][122],[123]$} \\
& {$[25],[48],[26],[20],[27]$} & \\
\hline $\begin{array}{l}\text { D3. Repol. dispersion } \\
\text { biophysical model }\end{array}$ & {$[29],[32],[34],[36]$} & {$[125],[126]$} \\
\hline
\end{tabular}




\section{Challenges AND FUtURE PERSPeCtives}

In the previous sections, a catalog of techniques for assessment of ventricular repolarization instability have been presented, with emphasis in the description of recent ECG processing developments.

It must be acknowledged, however, that most of the ECG features presented here are only currently used in the context of research studies and have not yet bridged the gap to clinical routine. For instance, the left ventricular ejection fraction is commonly the only metric considered to predict the benefit from an ICD. In other studies evaluating druginduced ECG changes, the QT interval, or the heart ratecorrected QT interval, is considered as the only marker to assess pro-arrhythmicity, despite its well-known limitations. Considering the six phases for the assessment of a novel risk marker, presented by Hlatky et al. (1. Proof of Concept, 2. Prospective validation, 3. Incremental value, 4. Clinical utility, 5. Clinical outcome and 6. Cost-effectiveness) [131], most of the risk indices presented in this review have at most been evaluated in studies that can be framed in phases 1 or 2 , performed in small, specific populations. For a risk marker to become part of established clinical protocols, it must prove not only to be capable of quantifying increased instability or risk, but its capacity has to be large enough to change clinical management procedures. This must be proved in largescale prospective trials, considering the cost-effectiveness ratio (including not only the cost of the test, but also the cost of patient management associated with its results). Bridging the gap from scientific research to the clinics is the main challenge in this area.

Considering that between $45-50 \%$ of SCD occur in the general population (without known heart disease) [132] one important challenge is to identify non-invasive markers that can be measured in routine tests such as 10-s ECG or standard Holter recordings. Taking into account the different etiology and complexity of factors involved in SCD, it is also important to consider the combination of different markers that have been proved to be associated with risk separately (possibly including also chemical and genetic biomarkers). While some single non-invasive indices may have in their own modest incremental value, combining indices quantifying different physiological phenomena may provide more robust prediction or help to discriminate between different types of negative outcomes [123]. In the same manner as standardized databases have helped in the development, validation and comparison of computerized methods in electrocardiography, the establishment of baseline risk models for common patient groups (including the general population) [133] and the availability of ECG and clinical data from well-designed riskstratification-based trials would lead to more focused efforts in risk stratification, accelerating the way to clinical impact.

Additionally, despite the widely acknowledged dynamic nature of repolarization instability and arrhythmias, most studies disregard this fact and consider just a measurement of a specific risk marker at a single time point, and analyze its ability to predict risk at some indefinite future time. Current challenges to advance in risk stratification studies include as- sessment of a marker's ability to track variations in arrhythmic risk (e.g. when monitoring the effects of drug treatments or the evolution of cardiac diseases) as well as determination of the time frame for arrhythmic complications that may lead to SCD. Also, risk stratification would benefit from the development of novel non-invasive ventricular repolarization markers, for which a better understanding of the interaction between substrate, triggers and modulators involved in SCD is fundamental. Multi-scale computational modeling combined with signal processing of cardiac electrical signals offer a powerful tool to gain insight into SCD mechanisms and can definitely help in the development of personalized non-invasive clinical markers.

\section{Conclusions}

In this work, a review of technologies developed to quantify ventricular repolarization dispersion from the ECG is presented. The review focuses on so-called physiologically-driven model-based signal processing approaches. Some methods are developed from basic descriptive ECG considerations, while others come from either simplified or detailed biophysical modeling of the ventricular myocardium. While some of the presented methods aim at capturing stationary features, others provide information regarding the dynamics of repolarization, typically following HR variations. In any case, the final objective of the presented methodologies is its clinical impact and this requires companion clinical studies where to assess their utility. We firmly believe that combining clinical, experimental and technological development plus the use of physiologicallydriven model-based signal processing approaches is a right path to pursue the limits of SCD prediction from the ECG.

\section{REFERENCES}

[1] P. W. Macfarlane and T. D. Veitch-Lawrie, Eds., Comprehensive Electrocardiology. Theory and Practique in Health Disease. Pergamon Press, 1989.

[2] E. Pueyo, R. Bailón, E. Gil, J. P. Martínez, and P. Laguna, "Signal processing guided by physiology: making the most of cardiorespiratory signals," IEEE Signal Proc. Mag., pp. 136-142, 2013.

[3] L. Sörnmo and P. Laguna, Bioelectrical signal processing in cardiac and neurological applications. Elsevier, Academic Press, 2005.

[4] J. Malmivuo and R. Plonsey, Principles and Applications of Bioelectric and Biomagnetic Fields. Oxford University Press, New York, 1995.

[5] V. Shusterman, R. Lampert, and B. London, "The many faces of repolarization instability: which one is prognostic?" J Electrocardiol, vol. 42, no. 6, pp. 511-516, Nov 2009.

[6] E. Pueyo, J. P. Martínez, and P. Laguna, "Cardiac repolarization analysis using the surface electrocardiogram," Philos. T. Roy. Soc. A., vol. 367, no. 1887, pp. 213-233, 2009.

[7] T. W. Smith and M. E. Cain, "Sudden cardiac death: epidemiologic and financial worldwide perspective," J. Interv. Card. Electrophysiol., vol. 17, pp. 199-203, 2006.

[8] AHA, "American Heart Association," www.americanheart.org, 2007.

[9] B. A. Koplan and W. G. Stevenson, "Ventricular tachycardia and sudden cardiac death," Mayo Clin Proc, vol. 84, no. 3, pp. 289-297, May 2009.

[10] J. A. Vasallo, D. M. Cassidy, K. E. Kindwall, F. E. Marchlinski, and M. E. Josephson, "Non uniform recovery of excitability in the left ventricle," Circulation, vol. 78, no. 6, pp. 1365-1372, 1988.

[11] P. Coumel, "Cardiac arrhythmias and the autonomic nervous system," J. Cardiovasc. Electrophysiol., vol. 4(3), pp. 338-355, 1993.

[12] A. van Oosterom, "The dominant T wave," J. Electrocardiology, vol. 37 (suppl.), pp. 193-197, 2004.

[13] V. Iyer, R. Mazhari, and R. Winslow, "Computational model of the human left ventricular epicardial myocyte.” Biophys. J., vol. 87, pp. 1507-1525, 2004. 
[14] K. H. ten Tusscher and A. Panfilov, "Alternans and spiral breakup in a human ventricular tissue model," Am. J. Physiol. Heart Circ. Physiol., vol. 291, pp. H1088-H1100, 2006.

[15] E. Grandi, F. S. Pasqualini, and D. M. Bers, "A novel computational model of the human ventricular action potential and Ca transient," $J$. Mol. Cell. Cardiol, vol. 48, pp. 112-121, 2010.

[16] J. Carro, J. F. Rodríguez, P. Laguna, and E. Pueyo, "A human ventricular cell model for investigation of cardiac arrhythmias under hyperkalaemic conditions," Phil. Trans. R. Soc. A, vol. 369, pp. 42054232, 2011

[17] T. OHara, L. Virág, A. Varró, and Y. Rudy, "Simulation of the undiseased human cardiac ventricular action potential: Model formulation and experimental validation," PLoS Comput Biol,, vol. 7, p. e1002061, 2011

[18] C. S. Kuo and B. Surawicz, "Ventricular monophasic action potential changes associated with neurogenic $\mathrm{T}$ wave abnormalities and isoproterenol administration in dogs," Am. J. Cardiol, vol. 38(2), pp. 170-177, 1976.

[19] C. E. Conrath and T. Opthof, "Ventricular repolarization: an overview of (patho)physiology, sympathetic effects and genetic aspects," Prog. Biophys. Mol. Biol., vol. 92(3), pp. 269-307, 2006.

[20] A. Mincholé, E. Pueyo, J. F. Rodríguez, E. Zacur, M. Doblaré, and P. Laguna, "Quantification of restitution dispersion from the dynamic changes of the T-wave peak to end, measured at the surface ECG," IEEE Trans. on Biomed. Eng., vol. 58,, no. 5, pp. 1172-1182, 2011.

[21] E. Carmeliet, "Action potential duration, rate of stimulation, and intracellular sodium," J. Cardiovasc. Electrocardiol., vol. 17(1), pp. S2-S7, 2006

[22] L. M. Hondeghem, L. Carlsson, and G. Duker, "Instability and triangulation of the action potential predict serious proarrhythmia, but action potential duration prolongation is antiarrhythmic," Circulation, vol. 103, pp. 2004-2013, 2001.

[23] S. M. Narayan, "T-wave alternans and the susceptibility to ventricular arrhythmias," J. Am. Coll. Cardiol., vol. 47, pp. 269-281, 2006.

[24] J. M. Pastore, S. D. Girouard, K. R. Laurita, F. G. Akar, and D. S. Rosenbaum, "Mechanism linking T-wave alternans to the genesis of cardiac fibrillation," Circulation, vol. 99(10), pp. 1385-1394, 1999.

[25] M. P. Nash, C. P. Bradley, P. M. Sutton, R. H. Clayton, P. Kallis, M. P. Hayward, and et al., "Whole heart action potential duration restitution properties in cardiac patients: a combined clinical and modelling study," Exp Physiol, vol. 91, no. 2, pp. 339-354, 2006.

[26] H. N. Pak, S. J. Hong, G. S. Hwang, H. S. Lee, S. W. Park, and J. C. Ahn, "Spatial dispersion of action potential duration restitution kinetics is associated with induction of ventricular tachycardia/fibrillation in humans." J Cardiovasc Electrophysiol, vol. 15, no. 12, pp. 1357-1363, 2004.

[27] Q. Lou, V. V. Fedorov, A. V. Glukhov, N. Moazami, V. G. Fast, and I. R. Efimov, "Transmural heterogeneity and remodeling of ventricular excitation-contraction coupling in human heart failure," Circulation, vol. 123 , no. 17 , pp. 1881-90, 2011.

[28] C. L. Lawrence, C. E. Pollard, T. G. Hammond, and J. P. Valentin, "Nonclinical proarrhythmia models: predicting Torsades de Pointes," J. Pharmacol. Toxicol. Methods, vol. 52), pp. 46-59, 2005.

[29] A. van Oosterom, "Genesis of the T wave as based on an equivalent surface source model," J. of Electrocardiol., vol. 34, pp. 217-227, 2001.

[30] D. B. Geselowitz, "On the theory of the electrocardiogram," P. IEEE, vol. 77, no. 6, pp. 857-876, 1989.

[31] W. Miller and D. Geselowitz, "Simulation studies of the electrocardiogram - Part I: The normal heart," Circ. Res., vol. 43, no. 2, pp. 301-315, 1978

[32] D. B. Geselowitz, "Description of cardiac sources in anisotropic cardiac muscle. application of bidomain model," J. Electrocardiol, vol. 25, no. 1, pp. 65-67, 1992.

[33] R. Plonsey and R. C. Barr, "Current flow patterns in two-dimensional anisotropic bisyncytia with normal and extreme conductivities," Biophys., vol. 45, no. 3, pp. 557-571, 1984.

[34] R. Sassi and L. T. Mainardi, "An estimate of the dispersion of repolarization times based on a biophysical model of the ECG," IEEE Trans. on Biomed. Eng., vol. 58, no. 12, pp. 3396-3405, 2011.

[35] A. van Oosterom, "The dominant T wave and its significance," $J$. Cardiovasc. Electrophysiol., vol. 14, pp. S180-S187, 2003.

[36] V. S. Chauhan, E. Downar, K. Nanthakumar, J. D. Parker, H. J. Ross, W. Chan, and P. Picton, "Increased ventricular repolarization heterogeneity in patients with ventricular arrhythmia vulnerability and cardiomyopathy: A human in vivo study." Am. J. Physiol. Heart Circ. Physiol., vol. 290, pp. 79-86, 2006
[37] M. Lemay, J. M. Vesin, A. van Oosterom, V. Jacquemet, and L. Kappenberger, "Cancellation of ventricular activity in the ECG: Evaluation of novel and existing methods," IEEE Trans. Biomed. Eng.,, vol. 54, no. 3, pp. 542-546, 2007.

[38] A. van Oosterom, "Singular value decomposition of the T wave: Its link with a biophysical model of repolarization," Int. J. Bioelectromagn.,, vol. 4, pp. 59-60, 2002.

[39] F. Castells, P. Laguna, L. Sörnmo, A. Bollmann, and J. Millet, "Principal component analysis in ECG signal processing," EURASIP Journal on Advances in Signal Processing, vol. doi:10.1155/2007/74580, 2007.

[40] L. T. Mainardi and R. Sassi, "Some theoretical results on the observability of repolarization heterogeneity on surface ECG," J. Electrocardiol., vol. 46, pp. 270-275, 2013.

[41] J. P. Martínez, R. Almeida, S. Olmos, A. P. Rocha, and P. Laguna, "A wavelet-based ECG delineator: Evaluation on standard databases," IEEE Trans. Biomed. Eng., vol. 51, no. 4, pp. 570-581, 2004.

[42] R. Almeida, S. Gouveia, A. P. Rocha, E. Pueyo, J. P. Martínez, and P. Laguna, "QT variability and HRV interactions in ECG: quantification and reliability," IEEE Trans. Biomed. Eng., vol. 53, pp. 1317-1329, 2006.

[43] C. P. Day, J. M. McComb, and R. W. F. Campbell, "QT dispersion: An indication of arrhythmia risk in patients with long QT intervals," Br. Heart J., vol. 1, pp. 335-343, 1990.

[44] M. Malik, B. Acar, Y. Gang, Y. G. Yap, K. Hnatkova, and A. Camm, "QT dispersion does not represent electrocardiographic interlead heterogeneity of ventricular repolarization," J. Cardiovas. Electrophysiol, vol. 11, pp. 835-843, 2000.

[45] M. S. Fuller, G. Sándor, B. Punske, B. Taccardi, R. S. MacLeod, P. R. Ershler, L. S. Green, and R. L. Lux, "Estimates of repolarization dispersion from electrocardiographic measurements." Circulation, vol 102, pp. 685-691, 2000.

[46] P. Arini, G. C. Bertrán, E. R. Valverde, and P. Laguna, "T-wave width as an index for quantification of ventricular repolarization dispersion: Evaluation in an isolated rabbit heart model," Biomed. Signal Proces., vol. 3, pp. 67-77, 2008.

[47] P. D. Arini, F. H. Baglivo, J. P. Martínez, and P. Laguna, "Evaluation of ventricular repolarization dispersion during acute myocardial ischemia: spatial and temporal ECG indices," Med. Biol. Eng. Comput., vol. 52, pp. 375-391, 2014

[48] C. Antzelevitch, W. Shimizu, G. Yan, S. Sicouri, J. Weissenburger, V. Nesterenko, A. Burashnikov, J. D. Diego, J. Saffitz, and G. Thomas, "The M cell: Its contribution to the ECG and to normal and abnormal electrical function of the heart," J. Cardiovasc. Electrophysiol., vol. 10, pp. 1124-1152, 1999.

[49] T. Opthof, R. Coronel, F. J. Wilms-Schopman, A. N. Plotnikov, I. N. Shlapakova, P. Danilo, M. R. Rosen, and M. J. Janse, "Dispersion of repolarization in canine ventricle and the electrocardiographic $\mathrm{T}$ wave: Tpe interval does not reflect transmural dispersion," Heart Rhythm, vol. 4, no. 3, pp. 341-348, 2007.

[50] P. Laguna, R. Jané, and P. Caminal, "Automatic detection of wave boundaries in multilead ECG signals: Validation with the CSE database," Comput. Biomed. Res., vol. 27, pp. 45-60, 1994.

[51] M. Noriega, J. P. Martínez, P. Laguna, R. Bailón, and R. Almeida, "Respiration effect on wavelet-based ECG T-wave end delineation strategies," IEEE Trans Biomed Eng, vol. 59, pp. 1818-1828, 2012.

[52] S. Priori, D. Mortara, C. Napolitano, L. Diehl, V. Paganini, F. Cantú, G. Cantú, and P. Schwartz, "Evaluation of the spatial aspects of Twave complexity in the long-QT syndrome," Circulation, vol. 96, pp. 3006-3012, 1997.

[53] B. Acar, G. Yi, K. Hnatkova, and M. Malik, "Spatial, temporal and wavefront direction characteristics of 12-lead T-wave morphology," Med. Biol. Eng. Comput., vol. 37, pp. 574-584, 1999.

[54] P. Langley, D. Bernardo, and A. Murray, "Quantification of T wave shape changes following exercise," Pace, vol. 25, pp. 1230-1234, 2002.

[55] D. di Bernado and A. Murray, "Computer model for study of cardiac repolarization," J. Cardiovasc Electrophysiol, vol. 11, pp. 895-899, 2000

[56] S. Ahnve and H. Vallin, "Influence of heart rate and inhibition of autonomic tone on the QT interval," Circulation, vol. 65, pp. 435-439, 1982.

[57] M. Malik, "Problems of heart rate correction in assessment of druginduced QT interval prolongation," J. Cardiovasc. Electrophysiol., vol. 12, pp. 411-420, 2001.

[58] Y. G. Yap and A. J. Camm, "Drug induced QT prolongation and Torsades de Pointes," Heart, vol. 89, pp. 1363-1372, 2003. 
[59] L. Toivonen, K. Helenius, and M. Viitasalo, "Electrocardiographic repolarization during stress from awakening on alarm call," J. Am. Coll. Cardiol., vol. 30, pp. 774-779, 1997.

[60] C. P. Lau, A. R. Freedman, S. Fleming, M. Malik, A. J. Camm, and D. E. Ward, "Hysteresis of the ventricular paced QT interval in response to abrupt changes in pacing rate," Cardiovasc. Res., vol. 22, pp. 67-72, 1988.

[61] E. Pueyo, Z. Husti, T. Hornyik, I. Baczk, P. Laguna, A. Varr, and B. Rodrguez, "Mechanisms of ventricular rate adaptation as a predictor of arrhythmic risk," Am J Physiol Heart Circ Physiol, vol. 289, pp. H1577-H1587, 2010.

[62] M. R. Franz, C. D. Swerdlo, L. B. Liem, and J. Schaefer, "Cycle length dependence of human action potential duration in vivo. effects of single extrastimuli, sudden sustained rate acceleration and deceleration, and different steady-state frequencies," J Clin Invest, no. 82, pp. 972-979, 1988.

[63] E. Pueyo, P. Smetana, P. Caminal, A. B. de Luna, M. Malik, and P. Laguna, "Characterization of QT interval adaptation to RR interval changes and its use as a risk-stratifier of arrhythmic mortality in amiodarone-treated survivors of acute myocardial infarction," IEEE Trans. Biomed. Eng., vol. 51), pp. 1511-1520, 2004.

[64] E. Pueyo, M. Malik, and P. Laguna, "A dynamic model to characterize beat-to-beat adaptation of repolarization to heart rate changes," Biomed. Signal Proces., vol. 3, pp. 29-43, 2008.

[65] A. Grom, T. S. Faber, M. Brunner, C. Bode, and M. Zehender, "Delayed adaptation of ventricular repolarization after sudden changes in heart rate due to conversion of atrial fibrillation. A potential risk factor for proarrhythmia?" Europace, vol. 7, pp. 113-121, 2005.

[66] P. Smetana, E. Pueyo, K. Hnatkova, V. Batchvarov, P. Laguna, and M. Malik, "Individual patterns of dynamic QT/RR relationship in survivors of acute myocardial infarction and their relationship to antiarrhythmic efficacy of amiodarone," J. Cardiovasc. Electrophysiol., vol. 15, pp. 1147-1154, 2004.

[67] W. Shimizu, T. Ohe, T. K. T, and K. Shimomura, "Differential response of QTU interval to exercise, isoproterenol, and atrial pacing in patients with congenital long QT syndrome," Pacing Clin. Electrophysiol., vol. 14, pp. 1966-1970, 1991.

[68] A. R. Magnano, S. Holleran, R. Ramakrishnan, J. A. Reiffel, and D. M. Bloomfield, "Autonomic nervous system influences on QT interval in normal subjects," J. Am. Coll. Cardiol., vol. 39, pp. 1820-1826, 2002.

[69] R. D. Berger, "QT variability," J. Electrocardiol., vol. 36, pp. 83-87, 2003

[70] R. D. Berger, E. K. Kasper, K. L. Baughman, E. Marban, H. Calkins, and G. F. Tomaselli, "Beat-to-beat QT interval variability: Novel evidence for repolarization lability in ischemic and nonischemic dilated cardiomyopathy," circulation, vol. 96, pp. 1557-1565, 1997.

[71] S. Cuomo, F. Marciano, M. L. Migaux, F. Finizio, E. Pezzella, M. A. Losi, and S. Betocchi, "Abnormal QT interval variability in patients with hypertrophic cardiomyopathy: can syncope be predicted?" $J$. Electrocardiol., vol. 37, pp. 113-119, 2004.

[72] L. Burattini and W. Zareba, "Time-domain analysis of beat-to-beat variability of repolarization morphology in patients with ischemic cardiomyopathy," J. Electrocardiol., vol. 32, pp. 166-172, 1999.

[73] J. P. Couderc, W. Zareba, L. Burattini, and A. J. Moss, "Beat-to-beat repolarization variability in LQTS patients with the SCN5A sodium channel gene mutation," Pacing Clin. Electrophysiol., vol. 22, pp. 1581-1592, 1999

[74] J. S. Perkimki, W. Zareba, A. Nomura, M. Andrews, E. S. Kaufman, and A. J. Moss, "Repolarization dynamics in patients with long QT syndrome," J. Cardiovasc. Electrophysiol., vol. 13, pp. 651-656, 2002.

[75] A. Porta, G. Girardengo, V. Bari, A. L. George Jr., P. A. Brink, A. Goosen, L. Crotti, and P. J. Schwartz, "Autonomic control of heart rate and QT interval variability influences arrhythmic risk in long QT syndrome type 1," J Am Coll Cardiol., vol. 65, no. 4, pp. 367-374, 2015.

[76] A. Porta, G. Baselli, E. Caiani, A. Malliani, F. Lombardi, and S. Cerutti, "Quantifying electrocardiogram RT-RR variability interactions," Med. Biol. Eng. Comput., vol. 36, pp. 27-34, 1998.

[77] V. K. Yeragani, R. Berger, N. Desai, K. J. Bar, P. Chokka, and M. Tancer, "Relationship between beat-to-beat variability of RT-peak and RT-end intervals in normal controls, patients with anxiety, and patients with cardiovascular disease," Ann. Noninvasive Electrocardiol., vol. 12, pp. 203-209, 2007.

[78] J. Castro-Hevia, C. Antzelevitch, F. Tornés, M. Dorantes, F. Dorticós, R. Zayas, M. Quiones, and Y. Fayad, "Tpeak-Tend and Tpeak-Tend dispersion as risk factors for ventricular tachycardia/ventricular fibril- lation in patients with the Brugada syndrome," J. Am. Coll. Cardiol., vol. 47, pp. 1828-1834, 2006

[79] W. L. Atiga, L. Fananapazir, D. McAreavey, H. Calkins, and R. D. Berger, "Temporal repolarization lability in hypertrophic cardiomyopathy caused by beta-myosin heavy-chain gene mutations," Circulation, vol. 101, pp. 1237-1242, 2000.

[80] W. L. Atiga, H. Calkins, J. H. Lawrence, G. F. Tomaselli, J. F. Smith, and R. D. Berger, "Beat-to-beat repolarization lability identifies patients at risk for sudden cardiac death," J. Cardiovasc. Electrophysiol., vol. 9, pp. 899-908, 1998.

[81] G. Kudaiberdieva, B. Gorenek, O. Goktekin, Y. Cavusoglu, A. Birdane, A. Unalir, N. Ata, and B. Timuralp, "Combination of QT variability and signal-averaged electrocardiography in association with ventricular tachycardia in postinfarction patients," J. Electrocardiol., vol. 36, pp. 17-24, 2003.

[82] C. Lerma, T. Krogh-Madsen, and L. Glass, "Stochastic aspects of cardiac arrhythmias," J. Stat. Phys., vol. 128, pp. 347-374, 2007.

[83] M. Lemay, E. de Lange, and J. P. Kucera, "Effects of stochastic channel gating and distribution on the cardiac action potential," J. Theor. Biol., vol. 281, no. 1, pp. 84-96, 2011.

[84] E. Pueyo, A. Corrias, L. Virág, N. Jost, T. Szél, A. Varró, N. Szentandra, P. P. Nanasi, K. Burrage, and B. Rodríguez, "A multiscale investigation of repolarization variability and its role in cardiac arrhythmogenesis," Biophys. J., vol. 101, no. 12, pp. 2892-2902, 2011.

[85] J. Heijman, A. Zaza, D. M. Johnson, Y. Rudy, R. L. M. Peeters, P. G. A. Volders, and R. L. Westra, "Determinants of beat-to-beat variability of repolarization duration in the canine ventricular myocyte: A computational analysis," PLOS Comput. Biol., vol. 9, no. 8, p. e1003202, 2013.

[86] B. Hanson, J. Gill, D. Western, M. Gilbey, J. Bostock, M. R. Boyett, H. Zhang, R. Coronel, and P. Taggart, "Cyclical modulation of human ventricular repolarization by respiration." Front Physiol, vol. 3, p. 379, 2012.

[87] P. D. Larsen, Y. C. Tzeng, P. Y. Sin, and D. C. Galletly, "Respiratory sinus arrhythmia in conscious humans during spontaneous respiration." Respir Physiol Neurobiol, vol. 174, pp. 111-118, 2010.

[88] P. Kohl, C. Bollensdorff, and A. Garny, "Effects of mechanosensitive ion channels on ventricular electrophysiology: experimental and theoretical models," Exp Physiol, vol. 91, pp. 307-321, 2006.

[89] V. Monasterio, P. Laguna, and J. P. Martínez, "Multilead analysis of Twave alternans in the ECG using principal component analysis," IEEE Trans Biomed Eng, vol. 57, no. 7, pp. 1880-1890, 2009.

[90] J. P. Martínez and S. Olmos, "Methodological principles of T wave alternans analysis. A unified framework," IEEE Trans. Biomed. Eng., vol. 52, no. 4, pp. 599-613, 2005

[91] R. L. Verrier, T. Klingeheben, M. Malik, N. El-Sherif, D. V. Exner, S. H. Hohnloser, T. Ikeda, J. P. Martínez, S. Narayan, T. Nieminen, and D. Rosenbaum, "Microvolt T-wave alternans physiological basis, methods of measurement, and clinical utility - Consensus Guideline by International Society for Holter and Noninvasive Electrocardiology," JACC, vol. 58, pp. 1309-1324, 2011.

[92] G. Turitto, E. B. Caref, G. El-Attar, M. Helal, A. Mohamed, R. P. Pedalino, and N. El-Sherif, "Optimal target heart rate for exerciseinduced T-wave alternans," Ann. Noninvasive Electrocardiol., vol. 6, no. 2, pp. 123-128, 2001.

[93] T. Klingenheben, P. Ptaszinski, and S. H. Hohnloser, "Quantitative assessment of microvolt T-wave alternans in patients with congestive heart failure," J. Cardiovasc. Electrophysiol., vol. 16, no. 6, pp. 620$624,2005$.

[94] S. M. Narayan and J. M. Smith, "Differing rate dependence and temporal distribution of repolarization alternans in patients with and without ventricular tachycardia." J. Cardiovasc. Electrophysiol., vol. 10, no. 1, pp. 61-71, 1999.

[95] J. P. Martínez, S. Olmos, G. Wagner, and P. Laguna, "Characterization of repolarization alternans during ischemia: time-course and spatial analysis." IEEE Trans. Biomed. Eng., vol. 53, no. 4, pp. 701-711, 2006.

[96] L. Burattini, S. Bini, and R. Burattini, "Repolarization alternans heterogeneity in healthy subjects and acute myocardial infarction patients," Med. Eng. Physics, vol. 34, pp. 305-312, 2012.

[97] D. M. Bloomfield, S. H. Hohnloser, and R. J. Cohen, "Interpretation and classification of microvolt T-wave alternans tests," J. Cardiovasc. Electrophysiol., vol. 13, pp. 502-512, 2002.

[98] M. Demidova, A. Martin-Yebra, J. P. Martínez, V. Monasterio, S. Koul, J. van der Pals, D. Romero, P. Laguna, D. Erlinge, and P. G. Platonov, "T-wave alternans in experimental myocardial infarction: time course and predictive value for the assessment of myocardial damage," $J$. Electrocardiol., vol. 46, pp. 263-269, 2013. 
[99] G. D. Clifford, F. Azuaje, and P. E. McSharry, Fundamental Algorithms. Artech House Publishing, 2006, ch. Pathophysiology Guided T-Wave Alternans Measurement, pp. 197-214.

[100] S. M. Narayan, J. M. Smith, B. D. Lindsay, M. E. Cain, and V. G. Dvila-Romn, "Relation of T-wave alternans to regional left ventricular dysfunction and eccentric hypertrophy secondary to coronary heart disease." American Journal of Cardiology, vol. 97, pp. 775-780, 2006.

[101] D. S. Rosenbaum, L. E. Jackson, J. M. Smith, H. Garan, J. N. Ruskin, and R. J. Cohen, "Electrical alternans and vulnerability to ventricular arrhythmias," N. Engl. J. Med., vol. 330, no. 4, pp. 235-241, 1994.

[102] B. D. Nearing and R. L. Verrier, "Modified moving average analysis of T-wave alternans to predict ventricular fibrillation with high accuracy," J. Appl. Physiol., vol. 92, no. 2, pp. 541-549, 2002

[103] M. Orini, B. Hanson, V. Monasterio, J. P. Martínez, M. Hayward, P. Taggart, and P. Lambiase, "Comparative evaluation of methodologies for T-wave alternans mapping in electrograms," IEEE Trans. Biomed. Eng., vol. 61, no. 2, pp. 308-316, 2014.

[104] V. Monasterio, G. D. Clifford, P. Laguna, and J. P. Martínez., "A multilead scheme based on periodic component analysis for $\mathrm{T}$ wave alternans analysis in the ECG." Ann. Biomed. Eng., vol. 38, no. 8, pp. 2532-2541, 2010

[105] R. Sameni, C. Jutten, and M. B. Shamsollahi, "Multichannel electrocardiogram decomposition using periodic component analysis," IEEE Trans Biomed Eng, vol. 55, no. 8, pp. 1935-1940, 2008.

[106] B. D. Nearing, P. H. Stone, and R. L. Verrier, "Frequency response characteristics required for detection of T-wave alternans during ambulatory ECG monitoring," Ann. Noninvasive Electrocardiol., vol. 1, pp. 103-112, 1996

[107] V. Monasterio, P. Laguna, I. Cygankiewicz, R. Vázquez, A. BayésGen'is, A. B. de Luna, and J. P. Martínez, “Average T-wave alternans activity in ambulatory ECG records predicts sudden cardiac death in patients with chronic heart failure," Heart Rhythm, vol. 9, no. 3, pp. $383-389,2012$

[108] V. Monasterio, J. P. Martínez, P. Laguna, S. McNitt, S. Polonsky, A. J. Moss, M. Haigney, W. Zareba, and J. P. Couderc, "Prognostic value of average T-wave alternans and QT variability for cardiac events in MADIT-II patients," J. Electrocardiol., vol. 46, pp. 480-486, 2013.

[109] R. Bailón, N. Garatachea, I. de la Iglesia, J. A. Casajús, and P. Laguna, "Influence of running stride frequency in heart rate variability analysis during treadmill exercise testing," IEEE Trans. Biomed. Eng., vol. 60, pp. 1796-1805, 2013.

[110] P. Albrecht, J. Arnold, S. Krishnamachari, and R. J. Cohen, "Exercise recordings for the detection of $\mathrm{t}$ wave alternans: Promises and pitfalls," J. Electrocardiol., vol. 29, pp. 46-51, 1996.

[111] R. Sassi and L. T. Mainardi, "T-wave alternans: lessons learned from a biophysical ECG model," J. Electrocardiol., vol. 45, pp. 566-570, 2012.

[112] C. Haarmark, C. Graff, M. P. Andersen, T. Hardahl, J. J. Struijk, E. Toft, and et al., "Reference values of electrocardiogram repolarization variables in a healthy population." J Electrocardiol, vol. 43, pp. 31-39, 2010

[113] P. Smetana, V. Batchvarov, K. Hnatkova, A. J. Camm, , and M. Malik, "Sex differences in the rate dependence of the $\mathrm{T}$ wave descending limb," Cardiovasc. Res., vol. 58, no. 3, pp. 549-554, 2013

[114] M. Zabel, R. Woosley, and M. R. Franz, "Is dispersion of ventricular repolarization rate dependent?" Pacing Clin Electrophysiol, vol. 20, pp. 2405-2411, 1997.

[115] R. Coronel, F. J. G. Wilms-Schopman, T. Opthof, and M. J. Janse, "Dispersion of repolarization and arrhythmogenesis," Heart Rhythm, vol. 6, no. 4, pp. 537-543, 2009.

[116] K. R. Laurita, S. D. Girouard, and D. S. Rosenbaum, "Modulation of ventricular repolarization by a premature stimulus: Role of epicardial dispersion of repolarization kinetics demonstrated by optical mapping of the intact guinea pig heart," Circ. Res., vol. 79, pp. 493-503, 1996.

[117] A. M. Yue, M. R. Franz, P. R. Roberts, and J. M. Morgan, "Global endocardial electrical restitution in human right and left ventricles determined by noncontact mapping," J. Amer. Coll. Cardiol., vol. 46, pp. 1067-1075, 2005.

[118] A. Mincholé, E. Zacur, E. Pueyo, and P. Laguna, "Modeling and quantification of repolarization feature dependency on heart rate." Method. Inform. Med., vol. 53, no. 4, pp. 324-328, 2014

[119] E. Heidenreich, J. M. Ferrero, M. Doblar, and J. F. Rodrguez, "Adaptive macro finite elements for the numerical solution of monodomain equations in cardiac electrophysiology," Ann. Biomed. Eng., vol. 38, pp. 2331-2345, 2010

[120] K. Gima and Y. Rudy, "Ionic current basis of electrocardiographic waveforms: A model study,", Circ. Res., vol. 90, pp. 889-896, 2002.
[121] A. Mincholé, A. Bueno-Orovio, P. Laguna, E. Pueyo, and B. Rodríguez, "ECG-based estimation of dispersion of APD restitution as a tool to stratify sotalol-induced arrhythmic risk,", J. Electrocardiol., vol. 48, pp. 867-873, 2015 .

[122] A. Mincholé, R. Ariga, S. Neubauer, H. Watkins, and B. Rodriguez, "Electrocardiographic abnormalities of hypertrophic cardiomyopathy," Computing in Cardiology, vol. 41, pp. 387-400, 2014.

[123] J. Ramírez, V. Monasterio, A. Mincholé, M. Llamedo, G. Lenis, I. Cygankiewicz, A. B. de Luna, M. Malik, J. P. Martínez, P. Laguna, and E. Pueyo, "Automatic SVM classification of sudden cardiac death and pump failure death from autonomic and repolarization ECG markers," J. Electrocardiol., vol. 48, no. 4, pp. 551-557, 2015.

[124] R. Sassi, L. Mainardi, P. Laguna, and J. F. Rodríguez, "Validation of the $\mathcal{V}$-index through finite element 2D simulations," vol. 40. Computing in Cardiology, 2013, pp. 337-340.

[125] R. Sassi, M. W. Rivolta, L. T. Mainardi, R. C. Reis, M. Rocha, A. Ribeiro, and F. Lombardi, "Spatial repolarization heterogeneity and survival in chagas disease." Methods Inf Med., vol. 53, no. 6, pp. 464468, 2014.

[126] M. W. Rivolta, L. T. Mainardi, and R. Sassi, "Quantification of ventricular repolarization heterogeneity during moxifloxacin or sotalol administration using $\mathcal{V}$-index," Physiol. Meas., vol. 36, no. 4, pp. 803211, 2015.

[127] S. B. Olsson, J. P. Amlie, and S. Yuan, Eds., Dispersion of Ventricular Repolarization. Futura Publishing, 2000

[128] M. P. Bonomini, S. J. Corizo, P. Laguna, and P. D. Arini, “2D ECG differences in frontal vs prefernetial planes in patients referred for percutaneous transluminal coronary angioplasty," Biomed. signal proces., vol. 11, pp. 97-106, 2014.

[129] M. L. Riccio, M. L. Koller, and R. F. Gilmour, "Electrical restitution and spatiotemporal organization during ventricular fibrillation," Circ. Res., vol. 84, pp. 955-963, 1999.

[130] R. J. Selvaraj, P. Picton, K. Nanthakumar, and V. S. Chauhan, "Steeper restitution slopes across right ventricular endocardium in patients with cardiomyopathy at high risk of ventricular arrhythmias," Amer. J. Physiol Heart Circ. Physiol., vol. 292, pp. 1262-1268, 2007.

[131] M. A. Hlatky, P. Greenland, D. K. Arnett, C. M. Ballantyne, M. H. Criqui, M. S. Elkind, A. S. Go, and et al., "Criteria for evaluation of novel markers of cariovascular risk. a scientific statement from the american heart association," Circulation, vol. 119, pp. 2408-2416, 2009.

[132] H. J. Wellens, P. J. Schwartz, F. W. Lindemans, A. E. Buxton, J. J. Goldberger, S. H. Hohnloser, and et al., "Riks stratification for suden cardiac death: current status and challenges for the future," European Heart Journal, vol. 35, no. 25, pp. 56-64, 2014.

[133] J. J. Goldberger, A. Basu, R. Boineau, A. E. Buxton, M. E. Cain, J. M. Canty, P. Chen, S. S. Chugh, and et al., "Risk stratification for sudden cardiac death. a plan for the future," Circulation, vol. 129, pp. 516-626, 2014.

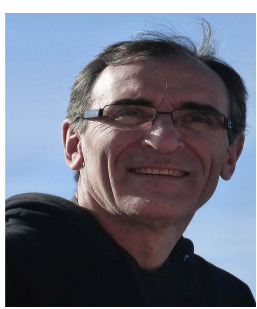

Pablo Laguna ( $\mathrm{M}^{\prime} 92$ - $\left.\mathrm{SM}^{\prime} 06\right)$ received the M.S degree in physics and the Ph.D. degree in physic science from the Science Faculty, University of Zaragoza, Spain, in 1985 and 1990, respectively. He is Full Professor of Signal Processing and Communications in the Department of Electrical Engineering at the Engineering School, and a researcher at the Aragón Institute for Engineering Research (I3A), both at University of Zaragoza, Spain. He is also member, and has served as scientific director (20112015), of the Spanish Center for Biomedical Engineering, Biomaterial and Nano-medicine Research CIBER-BBN.

His professional research interests are in Signal Processing, in particular applied to Biomedical applications. He has co-authored more than 120 research papers on this topic, over 250 international conference papers, and has advise $12 \mathrm{Ph} . \mathrm{D}$ Thesis. He is serving as predident the board of directors of Computing in Cardiology conference, editor of the digital signal processing journal (Eurasip), and of the Medical and Biological Engineering and Computing. He is also responsible of the Ph.D. program in Biomedical Engineering at Zaragoza University. He is, together with L. Sörnmo, the author of Bioelectrical Signal Processing in Cardiac and Neurological Applications, book (Elsevir, 2005) 


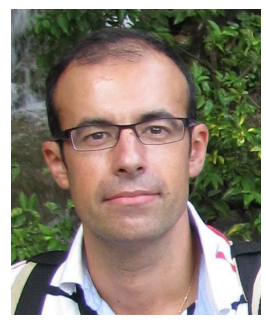

Juan Pablo Martinez Corts was born in Zaragoza, Aragón, in 1976. He received the M.Sc. degree in Telecommunication Engineering in 1999, and the $\mathrm{PhD}$ degree in Biomedical Engineering in 2005, both from the University of Zaragoza (UZ).

$\mathrm{He}$ is currently Associate Professor at the Department of Electrical Engineering and Communications and researcher at the Aragon Institute of Engineering Research at the University of Zaragoza (BSICoS Group), and is also affiliated with the Biomedical Research Networking center in Bioengineering, Biomaterials and Nanomedicine (CIBER-BBN), Spain. From 2010 to 2014, he has been the Coordinator of the M.Sc. Program on Biomedical Engineering. His research activities are focused on Statistical Signal Processing methods to obtain clinical information and risk indices from invasive and non-invasive cardiovascular signals.

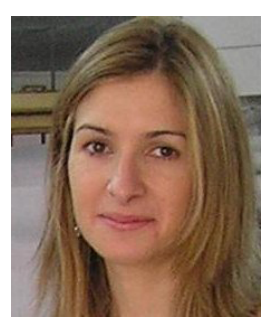

Esther Pueyo received B. Sc. and M.Sc. degrees in Mathematics in 1999 and 2001, respectively, as well as a M. Sc. degree in Communication and Information Technologies in 2002, from the University of Zaragoza, Spain. In 2006 she obtained her PhD degree in Biomedical Engineering with the European doctorate quality label.

She is currently Associate Professor at the University of Zaragoza and Researcher at the Biomedical Research Networking center in Bioengineering, Biomaterials and Nanomedicine (CIBER-BBN), Spain. Her research interests include signal processing of the electrocardiogram and electrophysiological modeling and simulation, with a focus on the analysis of ventricular repolarization for the investigation of cardiac arrhythmias. 